\title{
Coupled-cluster calculations of nucleonic matter
}

\author{
G. Hagen,${ }^{1,2}$ T. Papenbrock,${ }^{2,1}$ A. Ekström, ${ }^{3,4}$ K. A. Wendt,${ }^{2,1}$ \\ G. Baardsen, ${ }^{3}$ S. Gandolfi, ${ }^{5}$ M. Hjorth-Jensen, ${ }^{3,4,6}$ and C. J. Horowitz ${ }^{7}$ \\ ${ }^{1}$ Physics Division, Oak Ridge National Laboratory, Oak Ridge, TN 37831, USA \\ ${ }^{2}$ Department of Physics and Astronomy, University of Tennessee, Knoxville, TN 37996, USA \\ ${ }^{3}$ Department of Physics and Center of Mathematics for Applications, University of Oslo, N-0316 Oslo, Norway \\ ${ }^{4}$ National Superconducting Cyclotron Laboratory, Michigan State University, East Lansing, MI 48824, USA \\ ${ }^{5}$ Theoretical Division, Los Alamos National Laboratory Los Alamos, NM 87545 \\ ${ }^{6}$ Department of Physics and Astronomy, Michigan State University, East Lansing, MI 48824, USA \\ ${ }^{7}$ Indiana University, Bloomington, IN 47405, USA
}

Background: The equation of state (EoS) of nucleonic matter is central for the understanding of bulk nuclear properties, the physics of neutron star crusts, and the energy release in supernova explosions. Because nuclear matter exhibits a finely tuned saturation point, its EoS also constrains nuclear interactions.

Purpose: This work presents coupled-cluster calculations of infinite nucleonic matter using modern interactions from chiral effective field theory (EFT). It assesses the role of correlations beyond particle-particle and hole-hole ladders, and the role of three-nucleon-forces (3NFs) in nuclear matter calculations with chiral interactions.

Methods: This work employs the optimized nucleon-nucleon $(N N)$ potential NNLO $_{\text {opt }}$ at next-tonext-to leading-order, and presents coupled-cluster computations of the EoS for symmetric nuclear matter and neutron matter. The coupled-cluster method employs up to selected triples clusters and the single-particle space consists of a momentum-space lattice. We compare our results with benchmark calculations and control finite-size effects and shell oscillations via twist-averaged boundary conditions.

Results: We provide several benchmarks to validate the formalism and show that our results exhibit a good convergence toward the thermodynamic limit. Our calculations agree well with recent coupled-cluster results based on a partial wave expansion and particle-particle and hole-hole ladders. For neutron matter at low densities, and for simple potential models, our calculations agree with results from quantum Monte Carlo computations. While neutron matter with interactions from chiral EFT is perturbative, symmetric nuclear matter requires nonperturbative approaches. Correlations beyond the standard particle-particle ladder approximation yield non-negligible contributions. The saturation point of symmetric nuclear matter is sensitive to the employed $3 \mathrm{NFs}$ and the employed regularization scheme. $3 \mathrm{NFs}$ with nonlocal cutoffs exhibit a considerably improved convergence than their local cousins. We are unable to find values for the parameters of the short-range part of the local $3 \mathrm{NF}$ that simultaneously yield acceptable values for the saturation point in symmetric nuclear matter and the binding energies of light nuclei.

Conclusions: Coupled-cluster calculations with nuclear interactions from chiral EFT yield nonperturbative results for the EoS of nucleonic matter. Finite-size effects and effects of truncations can be controlled. For the optimization of chiral forces, it might be useful to include the saturation point of symmetric nuclear matter.

PACS numbers: 21.65.Mn, 21.65.Cd, 21.30.-x, 21.65.-f, 03.75.Ss, 26.60.-c, 26.60.Kp

\section{INTRODUCTION}

Bulk nucleonic matter is interesting for several reasons. The EoS of neutron matter, for instance, determines properties of supernova explosions [1], and of neutron stars [2], and it links the latter to neutron radii in atomic nuclei [8 10] and symmetry energy [11, 12. Likewise, the compressibility of nuclear matter is probed in giant dipole excitations [13, and the symmetry energy of nuclear matter is related to the difference between proton and neutron radii in atomic nuclei 14-16. The saturation point of nuclear matter determines bulk properties of atomic nuclei, and is therefore an important constraint for nuclear energy-density functionals and mass models (see, e.g., Refs. [17, 18]).

The determination and our understanding of the EoS for nuclear matter is intimately linked with our capability to solve the nuclear many-body problem. Here, correlations beyond the mean field play an important role. Theoretical studies of nuclear matter and the pertinent EoS span back to the very early days of nuclear manybody physics. Early computations are nicely described in the 1967 review by Day 19. These early calculations were performed using Brueckner-Bethe-Goldstone theory [20, 21], see Refs. [3, 22, 23] for recent reviews and developments. In these calculations, mainly particleparticle correlations were summed to infinite order. Other correlations were often included in a perturbative way. Coupled-cluster calculations of nuclear matter were performed already during the late 1970s and early 1980s [24, 25]. In recent years, there has been a considerable algorithmic development of first-principle methods 
for solving the nuclear many-body problem. A systematic inclusion of other correlations in a non-perturbative way are nowadays accounted for in Monte Carlo methods 26 30], self-consistent Green's function approaches [23, 31 34 and nuclear density functional theory [16, 18.

Similar progress has been made in the derivation of nuclear forces based on chiral EFT [35, 36. Nuclear Hamiltonians from chiral EFT are now used routinely in nucleonic matter calculations, with the 3NFs 37, 38 being front and center of many studies [32, 34, 39, 45. We note finally that there are also approaches to nucleonic matter based on lattice quantum chromodynamics [46].

In this work we study the EoS of nucleonic matter, using modern $N N$ interactions and 3NFs from chiral EFT, and an implementation of the coupled-cluster method [47, 48, that has become a standard in quantum chemistry [49, 50]. We employ a Cartesian momentum space basis with periodic boundary conditions, similar to the recent coupled-cluster based calculations of the electron gas [51, 52. Our calculations are based on coupled cluster with doubles (CCD) approximation [53 55]. This is the lowest-order truncation for closed-shell systems in a momentum-space basis, and we will also explore the role of selected triples clusters. We employ a recent parameterization [56] of the $N N$ force from chiral EFT at next-to-next-to-leading order, with inclusion of the $3 \mathrm{NF}$ that enters at the same chiral order.

This paper is organized as follows. In the next Section we present the coupled-cluster formalism for infinite matter that includes $3 \mathrm{NFs}$ and perturbative triples corrections. The calculations are performed in Cartesian coordinates with a discrete momentum basis and twisted periodic boundary conditions [57 $[59]$. This avoids the tedious partial-wave expansion of the nuclear forces, and it eases considerably the numerical evaluation of 3NFs. Averaging over twisted periodic boundary conditions minimizes finite-size effects and provides us with a good convergence towards the thermodynamic limit. Section II also presents computational results for finite-size effects and a few benchmark calculations. Our results for symmetric nuclear matter and pure neutron matter are presented in Sect. III. Concluding remarks are given in Sect. IV]

\section{METHOD}

In this Section we present the coupled-cluster formalism for infinite matter. We discuss the inclusion and treatment of $N N$ forces and 3NFs from chiral effective field theory (EFT), correlations up to three-particlethree-hole excitations and finite-size effects. Several benchmark calculations give us confidence in the validity of our approach.

\section{A. Interaction and model space}

Our Hamiltonian is

$$
H=T_{\text {kin }}+V_{N N}+V_{3 \mathrm{NF}} .
$$

Here, $T_{\text {kin }}$ denotes the kinetic energy, and $V_{N N}$ and $V_{3 \mathrm{NF}}$ denote the translationally invariant $N N$ interaction and $3 \mathrm{NF}$. The $N N$ interaction and $3 \mathrm{NF}$ are from chiral effective field theory 35,36 at next-to-next-to-leading order (NNLO). We employ the parameterization $\mathrm{NNLO}_{\text {opt }}$ for the $N N$ interaction [56], and the local 3NF [60]. This $3 \mathrm{NF}$ has a local regulator, i.e. the cutoff is in the momentum transfer, and thereby differs from implementations of the $3 \mathrm{NF}[38$ that employ the cutoff in the relative Jacobi momenta. We note that the numerical implementation of the $3 \mathrm{NF}$ in the discrete momentum basis is much simpler than in the harmonic oscillator basis commonly used for finite nuclei, because essentially no transformation of matrix elements is necessary. Nevertheless, the sheer number of matrix elements (and associated function calls) of the $3 \mathrm{NF}$ is huge, and this is computationally still a limiting factor.

For the model space, we choose a cubic lattice in momentum space with $\left(2 n_{\max }+1\right)^{3}$ momentum points. The spin (spin-isospin) degeneracy of each momentum point is $g_{s}=2\left(g_{s}=4\right)$ for pure neutron matter (nuclear matter). Thus, filling of the lattice yields shell closures for "Fermi spheres" with $g_{s} n$ fermions, and $n=1,7,19,27,33,57, \ldots$. We note that one could also use non-cubic lattices. Any periodic lattice permits one to implement momentum conservation exactly. For fixed particle number $A=g_{s} n$ and density $\rho=g_{s} k_{F}^{3} /\left(6 \pi^{2}\right)$ (or Fermi momentum $k_{F}$ ), one computes the volume of the cubic box $V=L^{3}=A / \rho$, and the box length $L$ that determines the lattice spacing $\Delta k=2 \pi / L$. We note that the computed results exhibit a dependence on the shell closure $n$. However, Subsection IID shows that shell effects and finite-size effects can be mitigated and controlled, and that the dependence on the parameter $n$ becomes very small.

The second parameter of our lattice is $n_{\max }$. We note that $n_{\max } \Delta k$ is the momentum cutoff of our singleparticle basis. One has to increase $n_{\max }$ until the computed results (e.g. the energy per nucleon) is practically independent of this parameter. For the results reported below we find that $n_{\max }=4$ is sufficient.

\section{B. Coupled-cluster theory for infinite systems}

In this Section we present the coupled-cluster equations for nucleonic matter. Our calculations of nucleonic matter are based on the recently optimized chiral nucleon-nucleon interaction at $\mathrm{NNLO}_{\text {opt }}$ [56] with the $3 \mathrm{NF}$ at the same chiral order. The low-energy constants (LECs) of the $3 \mathrm{NF}$ were determined by fitting the constants $c_{E}$ and $c_{D}$ to reproduce the experimental half-life 
and binding energy of the triton. The optimized $3 \mathrm{NF}$ LECs are $c_{E}=-0.389$ and $c_{D}=-0.39$. With these values the ${ }^{4} \mathrm{He}$ binding energy is $-28.47 \mathrm{MeV}$ 61. We employ single-particle states

$$
\left|\vec{p}, s_{z}, t_{z}\right\rangle \equiv|\mathbf{k}\rangle
$$

with momentum $\vec{k}$, spin projection $s_{z}$ and isospin projection $t_{z}$. Discrete values of the momentum variable $\vec{p}=\hbar \vec{k}$ result from periodic boundary conditions in a cubic box with length $L$, that is

$$
k_{n_{i}}=\frac{2 \pi n_{i}}{L}, n_{i}=0, \pm 1, \ldots \pm n_{\max }, i=x, y, z
$$

In this basis, the nuclear Hamiltonian with nucleonnucleon and three-nucleon interactions is

$$
\begin{aligned}
H & =\sum_{p q}\left\langle\mathbf{k}_{p}\left|T_{\mathrm{kin}}\right| \mathbf{k}_{q}\right\rangle a_{p}^{\dagger} a_{q} \\
& +\frac{1}{4} \sum_{p q r s}\left\langle\mathbf{k}_{p} \mathbf{k}_{q}\left|V_{\mathrm{NN}}\right| \mathbf{k}_{r} \mathbf{k}_{s}\right\rangle a_{p}^{\dagger} a_{q}^{\dagger} a_{s} a_{r} \\
& +\frac{1}{36} \sum_{p q r s t u}\left\langle\mathbf{k}_{p} \mathbf{k}_{q} \mathbf{k}_{r}\left|V_{3 \mathrm{NF}}\right| \mathbf{k}_{s} \mathbf{k}_{t} \mathbf{k}_{u}\right\rangle a_{p}^{\dagger} a_{q}^{\dagger} a_{r}^{\dagger} a_{u} a_{t} a_{s}
\end{aligned}
$$

The kinetic energy is diagonal in the discrete momentum basis $\left\langle\mathbf{k}_{p}\left|T_{\text {kin }}\right| \mathbf{k}_{q}\right\rangle=\frac{\hbar^{2}}{2 m} \vec{k}_{p}^{2} \delta_{p q}$. The operators $a_{p}^{\dagger}$ and $a_{p}$ create and annihilate a nucleon in state $\left|\mathbf{k}_{p}\right\rangle$, respectively.

The discrete momentum basis allows us to respect translational invariance of the $N N$ potential and the $3 \mathrm{NFs}$. Momentum is conserved, meaning that the twoand three-body matrix elements of the Hamiltonian (1) vanish unless

$$
\vec{k}_{p}+\vec{k}_{q}=\vec{k}_{r}+\vec{k}_{s}
$$

and

$$
\vec{k}_{p}+\vec{k}_{q}+\vec{k}_{r}=\vec{k}_{s}+\vec{k}_{t}+\vec{k}_{u}
$$

Note also that the chiral nucleon-nucleon and threenucleon interactions conserve the total isospin projection, but not the total spin projection.

In single-reference coupled-cluster theory the correlated wave-function is written in the form

$$
|\Psi\rangle=e^{T}\left|\Phi_{0}\right\rangle
$$

Here $\left|\Phi_{0}\right\rangle=\prod_{i=1}^{A} a_{i}^{\dagger}|0\rangle$ is a product state and serves as the reference. The cluster operator $T$ is a linear combination of $n$-particle- $n$-hole $(n p$ - $n h)$ excitation operators, i.e. $T=T_{1}+T_{2}+\ldots+T_{n}$. In the discretized momentum basis the reference state is the closed shell Fermi vacuum, and is obtained by filling the $A$ states with the lowest kinetic energy. We limit ourselves to spin saturated reference state, meaning that each momentum orbital of the reference state is doubly occupied. In this case the nuclear interaction does not induce $1 p$ - $1 h$ excitations of the reference state, and we have $T_{1}=0$. Thus, the cluster operator becomes

$$
T=\frac{1}{4} \sum_{i j a b}\left\langle\mathbf{k}_{a} \mathbf{k}_{b}|t| \mathbf{k}_{i} \mathbf{k}_{j}\right\rangle a_{a}^{\dagger} a_{b}^{\dagger} a_{j} a_{i}+\ldots
$$

Here and in what follows, indices $i, j, k, l(a, b, c, d)$ label occupied (unoccupied) states. Truncating $T$ at the $2 p$ $2 h$ excitation level $\left(T \approx T_{2}\right)$ gives the coupled-cluster doubles (CCD) approximation. The CCD energy and amplitude equations can be written in compact form

$$
\begin{aligned}
E_{\mathrm{CCD}} & =E_{0}+\left\langle\Phi_{0}\left|\bar{H}_{N}\right| \Phi_{0}\right\rangle, \\
0 & =\left\langle\Phi_{i j}^{a b}\left|\bar{H}_{N}\right| \Phi_{0}\right\rangle .
\end{aligned}
$$

Here

$$
\begin{aligned}
E_{0}= & \left\langle\Phi_{0}|H| \Phi_{0}\right\rangle \\
= & \sum_{i}\left\langle\mathbf{k}_{i}|f| \mathbf{k}_{i}\right\rangle+\frac{1}{2} \sum_{i, j}\left\langle\mathbf{k}_{i} \mathbf{k}_{j}|v| \mathbf{k}_{i} \mathbf{k}_{j}\right\rangle \\
& +\frac{1}{6} \sum_{i j k}\left\langle\mathbf{k}_{i} \mathbf{k}_{j} \mathbf{k}_{l}|w| \mathbf{k}_{i} \mathbf{k}_{j} \mathbf{k}_{l}\right\rangle
\end{aligned}
$$

is the vacuum expectation value (which in the case of no $1 p$ - $1 h$ corresponds to the Hartree-Fock energy), $\left|\Phi_{i j}^{a b}\right\rangle$ is a $2 p-2 h$ excitation of the reference state, and $\bar{H}_{N} \equiv$ $e^{-T} H_{N} e^{T}$ is the similarity transformation of the normalordered Hamiltonian

$$
\begin{aligned}
H_{N} & =\sum_{p q}\left\langle\mathbf{k}_{p}|f| \mathbf{k}_{q}\right\rangle: a_{p}^{\dagger} a_{q}: \\
& +\frac{1}{4} \sum_{p q r s}\left\langle\mathbf{k}_{p} \mathbf{k}_{q}|v| \mathbf{k}_{r} \mathbf{k}_{s}\right\rangle: a_{p}^{\dagger} a_{q}^{\dagger} a_{s} a_{r}: \\
& +\frac{1}{36} \sum_{p q r s t u}\left\langle\mathbf{k}_{p} \mathbf{k}_{q} \mathbf{k}_{r}|w| \mathbf{k}_{s} \mathbf{k}_{t} \mathbf{k}_{u}\right\rangle: a_{p}^{\dagger} a_{q}^{\dagger} a_{r}^{\dagger} a_{u} a_{t} a_{s}:
\end{aligned}
$$

Here $: a_{p}^{\dagger} \ldots a_{p^{\prime}} \ldots:$ is the normal ordered string of operators with respect to the reference state. The normalordered one-body operator is given in terms of the Fock matrix elements

$$
\begin{aligned}
\left\langle\mathbf{k}_{p}|f| \mathbf{k}_{q}\right\rangle & =\left\langle\mathbf{k}_{p}|t| \mathbf{k}_{q}\right\rangle+\sum_{i}\left\langle\mathbf{k}_{p} \mathbf{k}_{i}\left|V_{\mathrm{N} N}\right| \mathbf{k}_{q} \mathbf{k}_{i}\right\rangle \\
& +\frac{1}{2} \sum_{i j}\left\langle\mathbf{k}_{p} \mathbf{k}_{i} \mathbf{k}_{j}\left|V_{3 \mathrm{NF}}\right| \mathbf{k}_{q} \mathbf{k}_{i} \mathbf{k}_{j}\right\rangle
\end{aligned}
$$

The normal-ordered two-body operator has matrix elements

$$
\begin{aligned}
\left\langle\mathbf{k}_{p} \mathbf{k}_{q}|v| \mathbf{k}_{r} \mathbf{k}_{s}\right\rangle & =\left\langle\mathbf{k}_{p} \mathbf{k}_{q}\left|V_{\mathrm{NN}}\right| \mathbf{k}_{r} \mathbf{k}_{s}\right\rangle \\
& +\sum_{i}\left\langle\mathbf{k}_{p} \mathbf{k}_{q} \mathbf{k}_{i}\left|V_{3 \mathrm{NF}}\right| \mathbf{k}_{i} \mathbf{k}_{r} \mathbf{k}_{s}\right\rangle
\end{aligned}
$$

Finally, the normal-ordered three-body operator $w$ has matrix elements

$$
\left\langle\mathbf{k}_{p} \mathbf{k}_{q} \mathbf{k}_{r}|w| \mathbf{k}_{s} \mathbf{k}_{t} \mathbf{k}_{u}\right\rangle=\left\langle\mathbf{k}_{p} \mathbf{k}_{q} \mathbf{k}_{r}\left|V_{3 \mathrm{NF}}\right| \mathbf{k}_{s} \mathbf{k}_{t} \mathbf{k}_{u}\right\rangle
$$


In most of this work, we will neglect all elements of $w$ when solving the CCD equations. In this normalordered two-body approximation, the $3 \mathrm{NF}$ enters in the vacuum expectation value (4), the Fock matrix (6), and the normal-ordered two-body operator (7), but the threebody operator $w$ that changes the orbitals of all three nucleons is neglected.

We note that coupled-cluster theory with full inclusion of 3NFs was worked out in the singles and doubles approximation (CCSD) 62, and very recently with triples corrections included 63 .

For an efficient numerical implementation one writes the CCD equations (3) in a factorized (quasi-linear) form,

$$
\begin{aligned}
0 & =\left\langle\mathbf{k}_{a} \mathbf{k}_{b}|v| \mathbf{k}_{i} \mathbf{k}_{j}\right\rangle \\
& +P(a b) \sum_{c}\left\langle\mathbf{k}_{b}|\chi| \mathbf{k}_{c}\right\rangle\left\langle\mathbf{k}_{a} \mathbf{k}_{c}|t| \mathbf{k}_{i} \mathbf{k}_{j}\right\rangle \\
& -P(i j) \sum_{k}\left\langle\mathbf{k}_{k}|\chi| \mathbf{k}_{j}\right\rangle\left\langle\mathbf{k}_{a} \mathbf{k}_{b}|t| \mathbf{k}_{i} \mathbf{k}_{k}\right\rangle \\
& +\frac{1}{2} \sum_{c d}\left\langle\mathbf{k}_{a} \mathbf{k}_{b}|\chi| \mathbf{k}_{c} \mathbf{k}_{d}\right\rangle\left\langle\mathbf{k}_{c} \mathbf{k}_{d}|t| \mathbf{k}_{i} \mathbf{k}_{j}\right\rangle \\
& +\frac{1}{2} \sum_{k l}\left\langle\mathbf{k}_{a} \mathbf{k}_{b}|t| \mathbf{k}_{k} \mathbf{k}_{l}\right\rangle\left\langle\mathbf{k}_{k} \mathbf{k}_{l}|\chi| \mathbf{k}_{i} \mathbf{k}_{j}\right\rangle \\
& +P(i j) P(a b) \sum_{k c}\left\langle\mathbf{k}_{a} \mathbf{k}_{c}|t| \mathbf{k}_{i} \mathbf{k}_{k}\right\rangle\left\langle\mathbf{k}_{k} \mathbf{k}_{b}|\chi| \mathbf{k}_{c} \mathbf{k}_{j}\right\rangle \\
& +\frac{1}{2} P(i j) \sum_{c d k}\left\langle\mathbf{k}_{a} \mathbf{k}_{k} \mathbf{k}_{b}|w| \mathbf{k}_{i} \mathbf{k}_{c} \mathbf{k}_{d}\right\rangle\left\langle\mathbf{k}_{c} \mathbf{k}_{d}|t| \mathbf{k}_{k} \mathbf{k}_{j}\right\rangle \\
& -\frac{1}{2} P(a b) \sum_{c k l}\left\langle\mathbf{k}_{a} \mathbf{k}_{k} \mathbf{k}_{l}|w| \mathbf{k}_{i} \mathbf{k}_{c} \mathbf{k}_{j}\right\rangle\left\langle\mathbf{k}_{c} \mathbf{k}_{b}|t| \mathbf{k}_{k} \mathbf{k}_{l}\right\rangle .
\end{aligned}
$$

Here, $P(p q) \equiv 1-P_{p q}$ is an antisymmetrization operator, and we employed the intermediates

$$
\begin{aligned}
& \left\langle\mathbf{k}_{b}|\chi| \mathbf{k}_{c}\right\rangle=\left\langle\mathbf{k}_{b}|f| \mathbf{k}_{c}\right\rangle \\
& \quad-\frac{1}{2} \sum_{k l d}\left\langle\mathbf{k}_{b} \mathbf{k}_{d}|t| \mathbf{k}_{k} \mathbf{k}_{l}\right\rangle\left\langle\mathbf{k}_{k} \mathbf{k}_{l}|v| \mathbf{k}_{c} \mathbf{k}_{d}\right\rangle \\
& +\frac{1}{4} \sum_{e d k l}\left\langle\mathbf{k}_{k} \mathbf{k}_{l} \mathbf{k}_{b}|w| \mathbf{k}_{e} \mathbf{k}_{d} \mathbf{k}_{c}\right\rangle\left\langle\mathbf{k}_{e} \mathbf{k}_{d}|t| \mathbf{k}_{k} \mathbf{k}_{l}\right\rangle \\
& \left\langle\mathbf{k}_{k}|\chi| \mathbf{k}_{j}\right\rangle=\left\langle\mathbf{k}_{k}|f| \mathbf{k}_{j}\right\rangle \\
& +\frac{1}{2} \sum_{k c d}\left\langle\mathbf{k}_{k} \mathbf{k}_{l}|v| \mathbf{k}_{c} \mathbf{k}_{d}\right\rangle\left\langle\mathbf{k}_{c} \mathbf{k}_{d}|t| \mathbf{k}_{j} \mathbf{k}_{l}\right\rangle \\
& \quad+\frac{1}{4} \sum_{c d l n}\left\langle\mathbf{k}_{l} \mathbf{k}_{n} \mathbf{k}_{k}|w| \mathbf{k}_{c} \mathbf{k}_{d} \mathbf{k}_{j}\right\rangle\left\langle\mathbf{k}_{c} \mathbf{k}_{d}|t| \mathbf{k}_{l} \mathbf{k}_{n}\right\rangle \\
& \left\langle\mathbf{k}_{k} \mathbf{k}_{l}|\chi| \mathbf{k}_{i} \mathbf{k}_{j}\right\rangle=\left\langle\mathbf{k}_{k} \mathbf{k}_{l}|v| \mathbf{k}_{i} \mathbf{k}_{j}\right\rangle \\
& +\frac{1}{2} \sum_{c d}\left\langle\mathbf{k}_{k} \mathbf{k}_{l}|v| \mathbf{k}_{c} \mathbf{k}_{d}\right\rangle\left\langle\mathbf{k}_{c} \mathbf{k}_{d}|t| \mathbf{k}_{i} \mathbf{k}_{j}\right\rangle \\
& +\frac{1}{2} P(i j) \sum_{c d n}\left\langle\mathbf{k}_{n} \mathbf{k}_{k} \mathbf{k}_{l}|w| \mathbf{k}_{c} \mathbf{k}_{d} \mathbf{k}_{j}\right\rangle\left\langle\mathbf{k}_{c} \mathbf{k}_{d}|t| \mathbf{k}_{n} \mathbf{k}_{i}\right\rangle
\end{aligned}
$$

$$
\begin{aligned}
& \left\langle\mathbf{k}_{k} \mathbf{k}_{b}|\chi| \mathbf{k}_{c} \mathbf{k}_{j}\right\rangle=\left\langle\mathbf{k}_{k} \mathbf{k}_{b}|v| \mathbf{k}_{c} \mathbf{k}_{j}\right\rangle \\
& +\sum_{l d}\left\langle\mathbf{k}_{k} \mathbf{k}_{l}|v| \mathbf{k}_{c} \mathbf{k}_{d}\right\rangle\left\langle\mathbf{k}_{c} \mathbf{k}_{d}|t| \mathbf{k}_{l} \mathbf{k}_{j}\right\rangle \\
& -\frac{1}{2} \sum_{d l n}\left\langle\mathbf{k}_{l} \mathbf{k}_{k} \mathbf{k}_{n}|w| \mathbf{k}_{d} \mathbf{k}_{j} \mathbf{k}_{c}\right\rangle\left\langle\mathbf{k}_{d} \mathbf{k}_{b}|t| \mathbf{k}_{l} \mathbf{k}_{n}\right\rangle \\
& +\frac{1}{2} \sum_{d e l}\left\langle\mathbf{k}_{l} \mathbf{k}_{k} \mathbf{k}_{b}|w| \mathbf{k}_{d} \mathbf{k}_{e} \mathbf{k}_{c}\right\rangle\left\langle\mathbf{k}_{d} \mathbf{k}_{e}|t| \mathbf{k}_{l} \mathbf{k}_{j}\right\rangle,
\end{aligned}
$$

$$
\begin{aligned}
& \left\langle\mathbf{k}_{a} \mathbf{k}_{b}|\chi| \mathbf{k}_{c} \mathbf{k}_{d}\right\rangle=\left\langle\mathbf{k}_{a} \mathbf{k}_{b}|v| \mathbf{k}_{c} \mathbf{k}_{d}\right\rangle \\
& -\frac{1}{2} P(a b) \sum_{e k l}\left\langle\mathbf{k}_{k} \mathbf{k}_{l} \mathbf{k}_{b}|w| \mathbf{k}_{e} \mathbf{k}_{c} \mathbf{k}_{d}\right\rangle\left\langle\mathbf{k}_{e} \mathbf{k}_{a}|t| \mathbf{k}_{k} \mathbf{k}_{l}\right\rangle \text {. }
\end{aligned}
$$

In Eqs. (9, 10, 11, 12, 13) and (14) the numerically expensive sums that involve products of two-body operators can all be implemented efficiently as matrix-matrix multiplications. The momentum conservation reduces the computational cost of the CCD equations to $n_{\mathrm{o}} n_{\mathrm{u}}^{3}$, where $n_{\mathrm{o}}\left(n_{\mathrm{u}}^{3}\right)$ is the number of occupied (unoccupied) momentum states. This is a considerable reduction in computational cycles as compared to the normal cost of the CCD equations which is $n_{\mathrm{o}}^{2} n_{\mathrm{u}}^{4}$ [49, and similar to the reduction of computational cost achieved in the angular momentum coupled scheme 65, 66.

The coupled-cluster equations (9) are solved numerically by iteration and yield the matrix elements of $T_{2}$. The CCD energy (2) is given in algebraic form by

$$
E_{\mathrm{CCD}}=E_{0}+\frac{1}{4} \sum_{i j a b}\left\langle\mathbf{k}_{i} \mathbf{k}_{j}|v| \mathbf{k}_{a} \mathbf{k}_{b}\right\rangle\left\langle\mathbf{k}_{a} \mathbf{k}_{b}|t| \mathbf{k}_{i} \mathbf{k}_{j}\right\rangle .
$$

Below, we will also employ an approximation (denoted as $\left.\mathrm{CCD}_{\text {ladd }}\right)$ that only uses the particle-particle and holehole ladders in the CCD equations, i.e.

$$
\begin{aligned}
0 & =\left\langle\mathbf{k}_{a} \mathbf{k}_{b}|v| \mathbf{k}_{i} \mathbf{k}_{j}\right\rangle \\
& +P(a b) \sum_{c}\left\langle\mathbf{k}_{b}|f| \mathbf{k}_{c}\right\rangle\left\langle\mathbf{k}_{a} \mathbf{k}_{c}|t| \mathbf{k}_{i} \mathbf{k}_{j}\right\rangle \\
& -P(i j) \sum_{k}\left\langle\mathbf{k}_{k}|f| \mathbf{k}_{j}\right\rangle\left\langle\mathbf{k}_{a} \mathbf{k}_{b}|t| \mathbf{k}_{i} \mathbf{k}_{k}\right\rangle \\
& +\frac{1}{2} \sum_{c d}\left\langle\mathbf{k}_{a} \mathbf{k}_{b}|v| \mathbf{k}_{c} \mathbf{k}_{d}\right\rangle\left\langle\mathbf{k}_{c} \mathbf{k}_{d}|t| \mathbf{k}_{i} \mathbf{k}_{j}\right\rangle \\
& +\frac{1}{2} \sum_{k l}\left\langle\mathbf{k}_{a} \mathbf{k}_{b}|t| \mathbf{k}_{k} \mathbf{k}_{l}\right\rangle\left\langle\mathbf{k}_{k} \mathbf{k}_{l}|v| \mathbf{k}_{i} \mathbf{k}_{j}\right\rangle
\end{aligned}
$$

The $\mathrm{CCD}_{\text {ladd }}$ approximation was used in Ref. 67] within coupled-cluster theory, and a similar approximation was also employed in other computations of nucleonic matter, see, e.g., Refs. 40, 41.

Let us also discuss the inclusion of three-body clusters. When going beyond the CCD approximation and considering triples excitations, one might question whether the residual three-body part $w$ can safely be neglected. After all, three-body forces directly induce excitations of threebody clusters. Below we will include the residual part $w$ when considering contributions from triples excitations 
to the correlation energy, and study the accuracy of the normal-ordered two-body approximation in the presence of triples excitations in neutron and symmetric nuclear matter. Very recently, Binder et al. employed chiral interactions softened via the similarity renormalization group transformation [64, 68, studied the effect of triples corrections in the presence of $3 \mathrm{NFs}$ in nuclei such as ${ }^{16} \mathrm{O}$ and ${ }^{40} \mathrm{Ca}$, and found it to be small 63 .

The full inclusion of triples in the presence of threebody forces is demanding and computationally expensive. Some effects of triples can be included in the $\mathrm{CCD}(\mathrm{T})$ approximation 69 that we extend to 3NFs. In $\mathrm{CCD}(\mathrm{T})$ the triples excitation amplitude is approximated as

$$
\begin{aligned}
t_{i j k}^{a b c} & \equiv\left\langle\mathbf{k}_{a} \mathbf{k}_{b} \mathbf{k}_{c}|t| \mathbf{k}_{i} \mathbf{k}_{j} \mathbf{k}_{k}\right\rangle \\
& \approx\left\langle\Phi_{i j k}^{a b b}\left|(v+w)\left(1+\hat{T}_{2}\right)\right| \Phi_{0}\right\rangle / \epsilon_{a b c}^{i j k}
\end{aligned}
$$

Here

$$
\epsilon_{a b c}^{i j k} \equiv f_{i}^{i}+f_{j}^{j}+f_{k}^{k}-f_{a}^{a}-f_{b}^{b}-f_{c}^{c} .
$$

The $\mathrm{CCD}(\mathrm{T})$ correction to the energy is

$$
\Delta E_{\mathrm{CCD}(\mathrm{T})}=\frac{1}{36} \sum_{i j k a b c}\left|t_{i j k}^{a b c}\right|^{2} / \epsilon_{a b c}^{i j k} .
$$

Employing the triples amplitude 116 with the inclusion of $w$ yields the energy correction $\Delta E_{\mathrm{CCD}(\mathrm{T})}$. We also consider the following approximations. Neglecting the residual three-body part $w$ yields the normal-ordered two-body approximation to the $\mathrm{CCD}(\mathrm{T})$ energy correction, denoted as $\Delta E_{\mathrm{CCD}(\mathrm{T}: \mathrm{w}=0)}$. Omitting the term $w T_{2}$ in Eq. (16) gives the energy correction $\Delta E_{\mathrm{CCD}\left(\mathrm{T}: w T_{2}=0\right)}$. Note that the numerically expensive term $w T_{2}$ in Eq. (16) consist of three distinct diagrams in which one sums over $p p, h h$ and $p h$ intermediate states, respectively. Below we will investigate the contributions of these three diagrams to the $\mathrm{CCD}(\mathrm{T})$ energy correction in neutron and symmetric nuclear matter.

\section{Ladder approximation in a partial-wave basis}

In Ref. 67, the ladder approximation of the coupledcluster equations for nuclear matter is presented in an alternative formulation. Historically, the equations for nuclear matter, for example in the hole-line approximation [70, have often been expressed explicitly in a partial-wave basis [71 73]. Similarly, in the method presented in Ref. [67], the ladder approximation is formulated in a partial-wave basis, assuming that the thermodynamic limit is reached and therefore using integrals over relative and center-of-mass momenta. In the partialwave expanded equations, the Pauli exclusion operators are treated exactly, using a technique introduced for the Brueckner-Hartree-Fock approximation by Suzuki et al. [73. Apart from the truncation in partial waves, the only approximation in this method is in the single-particle potentials, where an angular-average approximation was used for the laboratory momentum argument [67, 74].

\section{Finite size effects}

We would like to quantify the error due to finite size effects and the accuracy of our coupled-cluster calculations of neutron and nuclear matter. Using periodic boundary conditions (PBC) one could increase the number of particles in the box until convergence to the thermodynamic limit is reached. However, due to variations of the shell effects at different closed shell configurations, there is no guarantee that increasing the number of particles will lead to a systematic and smooth convergence to the thermodynamic limit. Furthermore, the computational cost of many-body methods such as the AFDMC and coupled-cluster methods increases rapidly with increasing particle number, and one would therefore like to employ a method that controls finite size effects already for modest particle numbers. This can be achieved with averaging over phases of Bloch waves that correspond to different boundary conditions [57 59].

Consider a free particle in a box of size $L$ subject to twisted boundary condition, that is, the wave function with momentum $k$ fulfills the condition for so-called Bloch waves, namely, $\psi_{k}(x+L)=e^{i \theta} \psi_{k}(x)$. By averaging over the twist angle $\theta$, shell effects can be eliminated for free Fermi systems [57, and they are much suppressed for interacting systems [58, 59. In this way, one obtains a much more systematic and smooth convergence towards the thermodynamic limit. The twisted boundary conditions are defined by

$$
k_{n_{i}}=\frac{\left(2 \pi n_{i}+\theta_{i}\right)}{L}, n_{i}=0, \pm 1, \ldots \pm n_{\max }, i=x, y, z,
$$

with the twist angle $\theta \in[0, \pi]$ for systems with timereversal invariance [59]. This amounts to letting the particles pick up a complex phase when they wrap around the boundary of the cubic box. By integrating or averaging over a finite number of twists in each $x, y, z$ direction we obtain the twist-averaged boundary conditions (TABC). In our implementation of TABC we integrate over the twist angles $\theta$ using a finite number of GaussLegendre quadrature points in $[0, \pi]$. Note that $\theta=0$ $(\theta=\pi)$ corresponds to (anti-)periodic boundary conditions.

In order to quantify the finite size effects using PBC and TABC we compute the kinetic and potential energy contribution to the Hartree-Fock energy for several closed shell configurations ranging from tens to several hundreds of nucleons, and compare with the thermodynamic limit for these quantities. In Fig. 1 we show the relative error of the kinetic energy in pure neutron matter for the Fermi momentum $k_{F}=1.6795 \mathrm{fm}^{-1}$ computed using standard PBC and TABC. We used 10 Gauss-Legendre points for the twist angle $\theta_{i}$ of the $i=x, y, z$ direction in the integration interval $[0, \pi]$. Clearly, we obtain a much faster and smoother convergence to the thermodynamic limit using TABC. Generally we get about an order of magnitude reduction in the relative error when using TABC as compared to PBC. Finite size effects are particularly 
small for PBC and $N=66$ neutrons. This was also seen in AFDMC calculations 27.

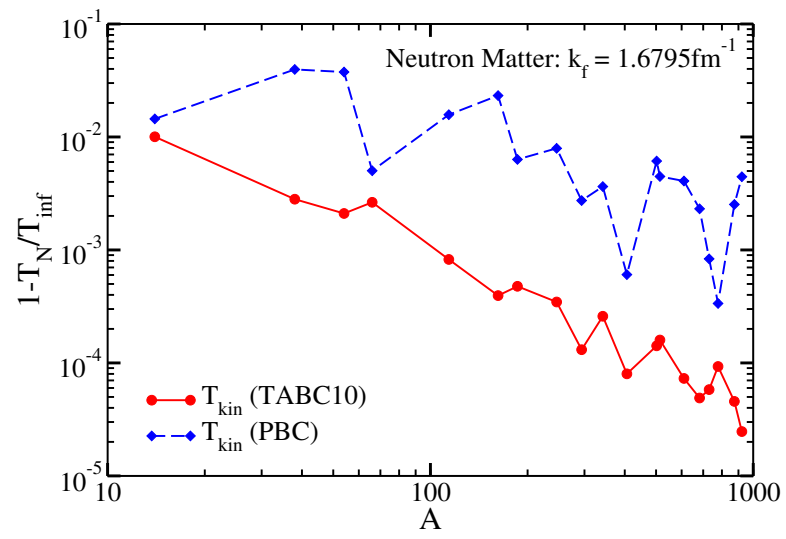

FIG. 1. (Color online) Relative finite-size corrections for the kinetic energy in pure neutron matter at the Fermi momentum $k_{F}=1.6795 \mathrm{fm}^{-1}$ vs. the neutron number $A$. TABC10 are twist-averaged boundary conditions with 10 Gauss-Legendre points in each spatial direction.

Figure 2 shows the relative error of the potential energy to the Hartree-Fock energy in pure neutron matter for the Fermi momentum $k_{F}=1.6795 \mathrm{fm}^{-1}$ computed with TABC. We compute the potential energy from $\mathrm{NNLO}_{\mathrm{opt}}$ and from the Minnesota potential. We see that the finite size effects in the potential energy are comparable to the finite size effects in the kinetic energy shown in Fig. 1. We note that finite size effects vanish as the power law $A^{-1.56}$ in the neutron number $A$.

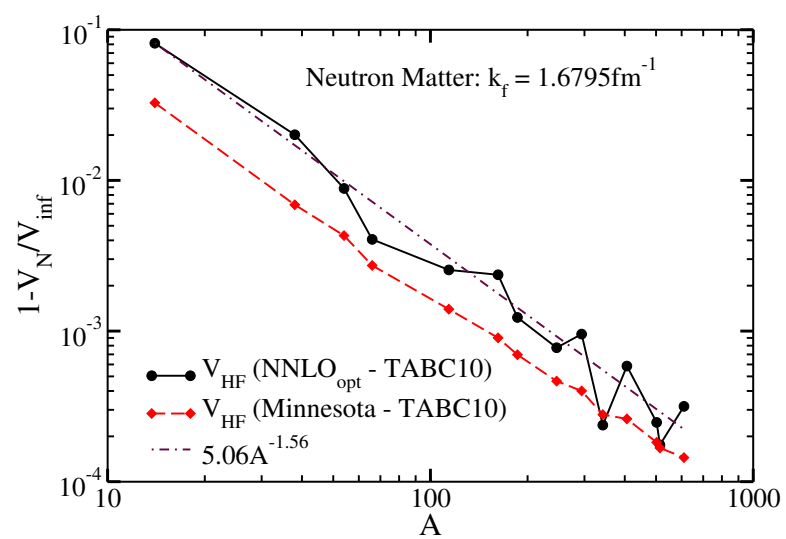

FIG. 2. (Color online) Relative finite-size corrections for the Hartree-Fock energy of the $\mathrm{NNLO}_{\mathrm{opt}}$ (full line) and Minnesota (dashed line) potentials in pure neutron matter at the Fermi momentum $k_{F}=1.6795 \mathrm{fm}^{-1}$ vs. the neutron number $A$. TABC10 are twist-averaged boundary conditions with 10 Gauss-Legendre points in each spatial direction. The dasheddotter line shows a power law fit to the $\mathrm{NNLO}_{\text {opt }}$ results.

Finally, we would also like to assess the finite-size effects in symmetric nuclear matter. In Fig. 3 we show the relative error of the potential energy to the Hartree-Fock energy in symmetric nuclear matter for the Fermi momentum $k_{F}=1.6 \mathrm{fm}^{-1}$ computed using $\mathrm{PBC}$ and TABC. We consider the Hartree-Fock potential energy contribution from the nucleon-nucleon interaction $\mathrm{NNLO}_{\text {opt }}$ and the $3 \mathrm{NF}$ at order NNLO separately. In particular it is seen that the relative error in the potential energy contribution from the $3 \mathrm{NF}$ is about an order of magnitude smaller than the relative error coming from the nucleonnucleon interaction alone using both PBC and TABC. In the case of symmetric nuclear matter there is no systematic convergence trend using $\mathrm{PBC}$, and for 132 nucleons the relative error for $\mathrm{PBC}$ is around $\sim 4 \%$, while using TABC the error is reduced to $\sim 1 \%$. It is interesting to note that finite size effects for $\mathrm{NNLO}_{\text {opt }}$ with TABC decrease as $A^{-1.59}$ with increasing nucleon number $A$. This exponent is similar to the exponent found in neutron matter (see Fig. 2).

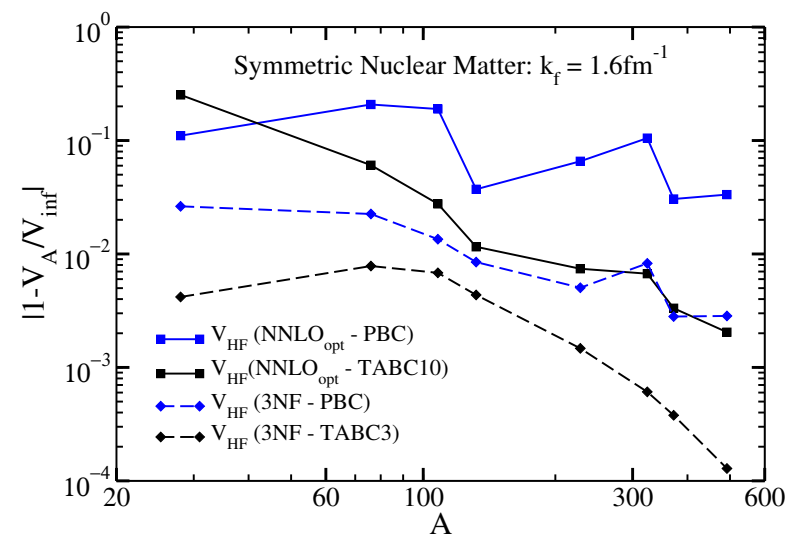

FIG. 3. (Color online) Relative finite-size corrections for the Hartree-Fock energy of the $N N$ potential $\mathrm{NNLO}_{\text {opt }}$ and the $3 \mathrm{NF}$ potential in symmetric nuclear matter at the Fermi momentum $k_{F}=1.6 \mathrm{fm}^{-1}$ vs. the nucleon number $A$. PBC: periodic boundary conditions. TABC10 and TABC3 are twistaveraged boundary conditions with 10 and 3 Gauss-Legendre points in each spatial direction, respectively.

Coupled-cluster calculations of nucleonic matter using $\mathrm{TABC}$ are very expensive. Using 10 twist angles in each direction requires $10^{3}$ coupled-cluster calculations, although symmetry considerations can reduce this number considerably. In Ref. [59] it was shown that one can find a specific choice of twist angles (known as special points), in which the Hartree-Fock energy exactly corresponds to the Hartree-Fock energy in the thermodynamic limit. In the following we compute these special points for neutron and nuclear matter using both $N N$ interactions and $3 \mathrm{NFs}$, and compare with calculations using $\mathrm{PBC}$ and TABC.

\section{E. Benchmarks}

It is interesting to compare the results for various boundary conditions with the infinite matter results by 
Baardsen et al. 67. Figure 4 shows the $\mathrm{CCD}_{\text {ladd }}$ results for neutron matter computed with the nucleon-nucleon potential $\mathrm{NNLO}_{\mathrm{opt}}$. In a finite system, the neutron number $N=66$ is very close to the infinite matter results for both periodic and twist-averaged boundary conditions.

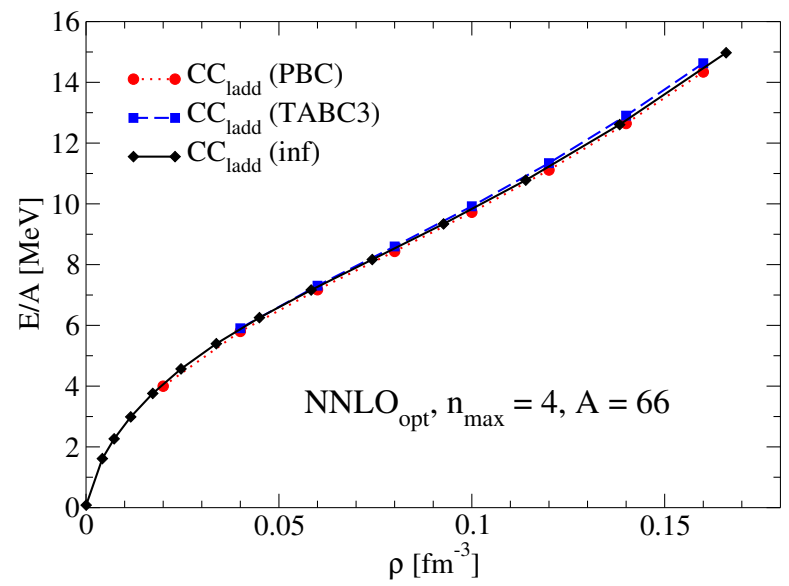

FIG. 4. (Color online) Energy per particle of neutron matter for $\mathrm{NNLO}_{\text {opt }}$ computed in the $\mathrm{CCD}_{\text {lad }}$ approximation with periodic boundary conditions (circles), twist-averaged boundary conditions (squares), and for infinite matter (diamonds). The latter results are from Ref. [56]. The calculations used $A=66$ neutrons and $n_{\max }=4$.

For symmetric nuclear matter, the CCD results are more sensitive to the choice of the boundary conditions, with results shown in Fig. 5. At higher Fermi momenta $\left(k_{F}>1.6 \mathrm{fm}^{-1}\right)$, the energy per nucleon for periodic boundary conditions differs by $\sim 0.5 \mathrm{MeV}$ from the result obtained with twist-averaged boundary conditions. A calculation with a special point in the twist is very close to the twist-averaged results. However, for Fermi momenta $k_{F}<1.6 \mathrm{fm}^{-1}$, the difference between the PBC and TABC is less than $200 \mathrm{keV}$ per nucleon.

Figure 6 compares nuclear matter results calculated in the ladder approximation with the $\mathrm{CC}_{\text {ladd }}$ of Ref. 67. The latter were obtained by taking the thermodynamic limit in the relative and center-of mass frame and by summing over partial waves. The summation over intermediate particle-particle and hole-hole configurations is performed with an exact Pauli operator, while the singleparticle energies are computed using an angle-averaging procedure, see Ref. 67] for further details. For these results, the angle-average approximation, together with a truncation in the number of partial waves included, represent the sources of possible errors in the thermodynamic limit. It is therefore very satisfactory that the results from different methods are close to each other.

Let us also consider a simple potential model and benchmark the results of our coupled-cluster calculations against virtually exact results from the auxiliary field diffusion Monte Carlo (AFDMC) method [75]. The Minnesota potential 76 is a semi-realistic nucleon-nucleon interaction that can be solved accurately with AFDMC.

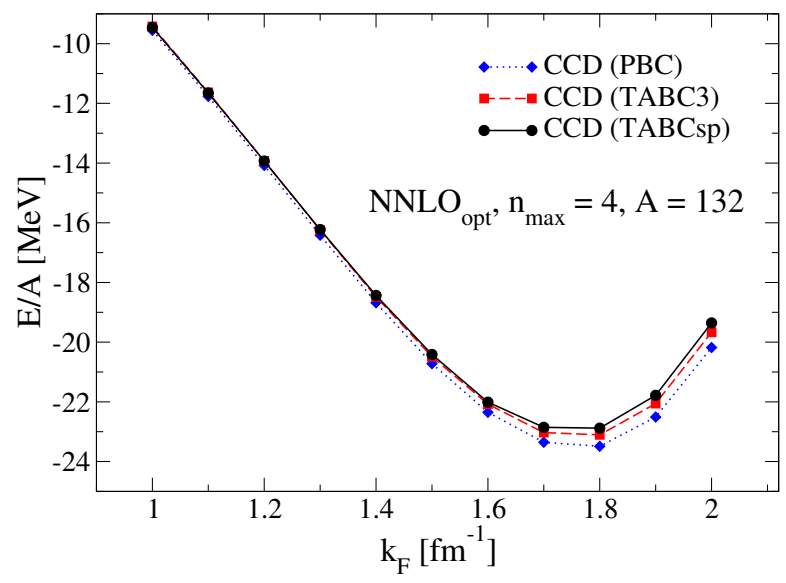

FIG. 5. (Color online) Energy per particle of symmetric nuclear matter for $\mathrm{NNLO}_{\text {opt }}$ computed in the CCD approximation with periodic boundary conditions (diamonds), twistaveraged boundary conditions (squares), and with a special point and twisted boundary conditions (circles). The calculations used $A=132$ nucleons and $n_{\max }=4$.

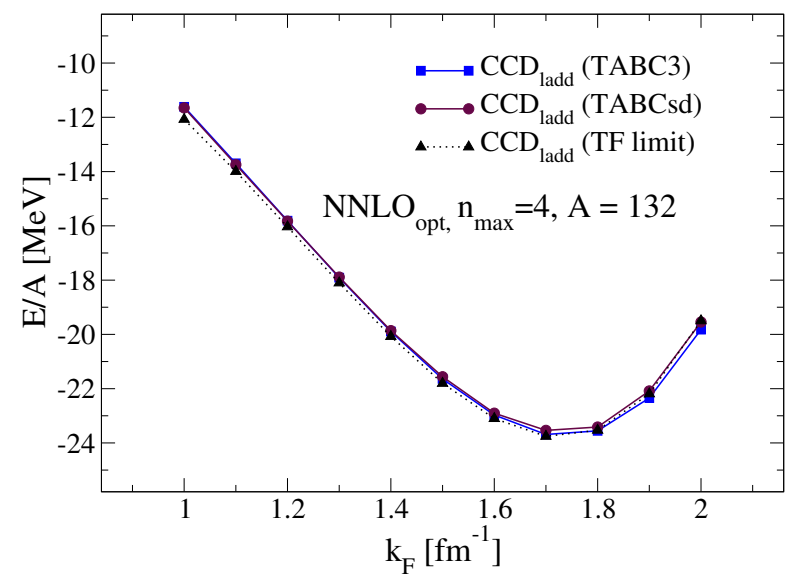

FIG. 6. (Color online) Energy per particle of symmetric nuclear matter for $\mathrm{NNLO}_{\text {opt }}$ computed in the $\mathrm{CCD}_{\text {ladd }}$ approximation in the thermodynamic limit using partial-wave expansion (triangles) (partly adapted from Ref. 67), with twist averaged boundary conditions (squares), and with a special point and twisted boundary conditions (circles). The calculations used $A=132$ nucleons and $n_{\max }=4$.

It depends only on the relative momenta and spin, but lacks spin-orbit or tensor contributions. The matrix elements of this potential are real numbers. For the benchmark we employ periodic boundary conditions, $A=66$ neutrons, and $n_{\max }=6$.

Figure 7 compares the energy per neutron of our lattice CCD results (circles), and our $\mathrm{CCD}_{\text {ladd }}$ in the thermodynamic limit, see Ref. 67, to the AFDMC benchmark. Overall, the agreement is good between all methods. As expected, the CCD results are more accurate than the $\mathrm{CCD}_{\text {ladd }}$ approximation.

Finally, we turn to $3 \mathrm{NFs}$. The inclusion of $3 \mathrm{NFs}-$ even in the normal-ordered approximation - is still nu- 


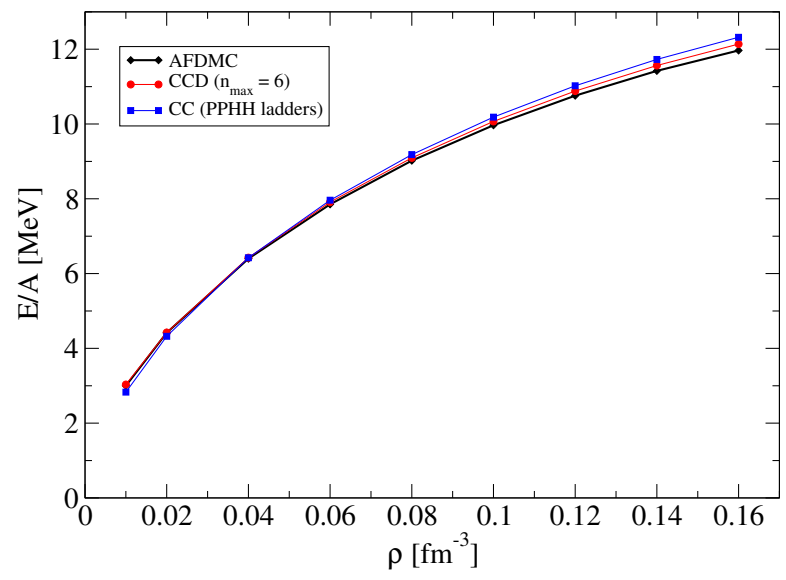

FIG. 7. (Color online) Energy per particle of neutron matter, computed with the Minnesota potential. Diamonds: AFDMC, circles: CCD, squares: CCD limited to $p p$ and $h h$ ladders.

merically expensive due to the large number of required matrix elements. We also study different approximations for $3 \mathrm{NFs}$, and compare the results for symmetric nuclear matter when 3NFs only enter in the normal-ordered approximation as 0-body, 1-body, or up to 2-body forces. Figure 8 clearly shows that normal-ordered 2-body forces are relevant.

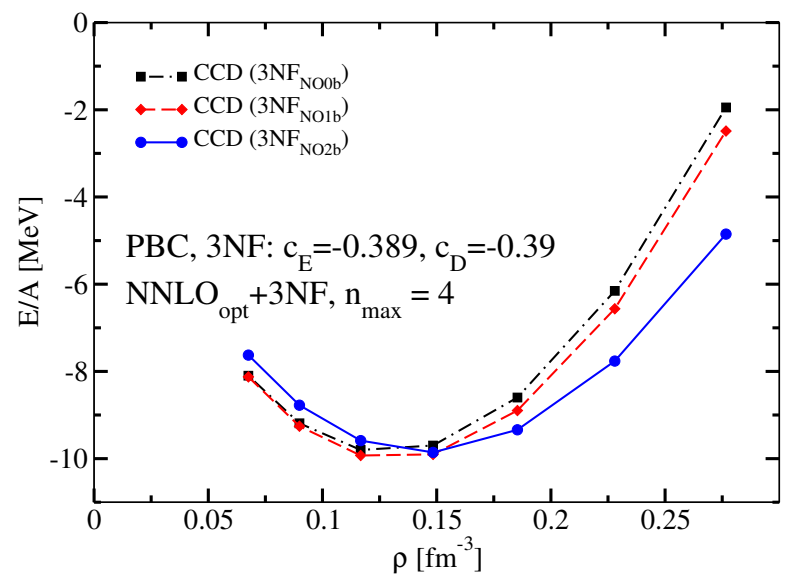

FIG. 8. (Color online) Energy per particle of symmetric nuclear matter computed in the CCD approximation with $\mathrm{NNLO}_{\text {opt }}+3 \mathrm{NF}\left(\mathrm{c}_{\mathrm{E}}=-0.389, \mathrm{c}_{\mathrm{D}}=-0.39\right)$. The $3 \mathrm{NF}$ is included in the 0-body (black dashed-dotted line), 1-body (red dashed line), and in the two-body (blue solid line) normalordered approximations. The calculations used $A=132 \mathrm{nu}-$ cleons and $n_{\max }=4$.

\section{RESULTS FOR CHIRAL INTERACTIONS}

In this Section, we present our results for coupledcluster computations of neutron matter and symmetric nuclear matter. As shown in the previous Section, the finite size effects (and the differences between $\mathrm{PBC}$ and TABC) are small for $A=66$ neutrons and $A=132 \mathrm{nu}-$ cleons when calculating neutron matter and symmetric nuclear matter, respectively. For this reason, many of the expensive calculations involving 3NFs are only performed with $\mathrm{PBC}$ at these specific particle numbers.

\section{A. Neutron matter}

Figure 9 shows the energy per neutron as a function of density based on $N N$ interactions alone and compares various many-body methods. The employed $N N$ interaction $\mathrm{NNLO}_{\text {opt }}$ is perturbative in neutron matter, with second-order many-body perturbation theory (MBPT2), $\mathrm{CCD}$ and $\mathrm{CCD}_{\text {ladd }}$ giving similar results that differ by less than $1 \mathrm{MeV}$ per neutron at nuclear saturation density.

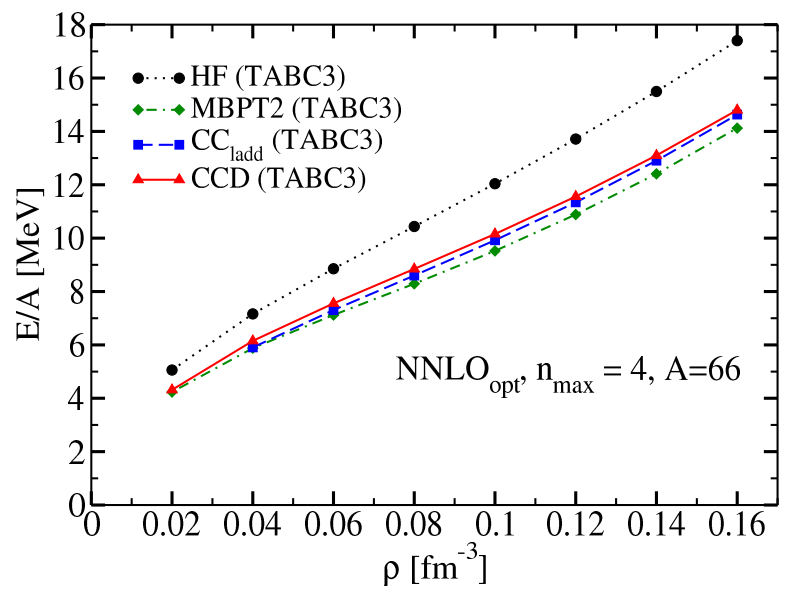

FIG. 9. (Color online) Energy per particle in neutron matter with $\mathrm{NNLO}_{\text {opt }}$ (NN only). The black dashed line is Hartree-Fock (HF), the green dashed-dotted line is secondorder many-body perturbation theory (MBPT2), the blue dashed line is coupled-cluster doubles ladder approximation $\left(\mathrm{CCD}_{\text {ladd }}\right)$, and the red solid line is coupled-cluster doubles (CCD). The calculations used $A=66$ neutrons, $n_{\max }=4$, and TABC3.

Figure 10 shows the effect of 3NFs in CCD calculations of the EoS for neutron matter. We consider several approximations involving 3NFs, and it is seen that they yield very similar results. We note that three-nucleon forces act repulsively. The results for neutron matter reported here are consistent with the recent calculations of Krueger et al. 44, and our results for the EoS fall within their NNLO uncertainty band. The CCD calculation that includes the normal-ordered 3NFs is shown as diamonds. Triples corrections that are limited to the inclusion of up to two-body terms from the normal-ordered $3 \mathrm{NF}$ are shown as circles, while triples corrections that include also the residual $3 \mathrm{NF}$ are shown as squares. For neutron matter, the effects of triples are small and account for about $0.3 \mathrm{MeV}$ per neutron at high densities, 
and the residual 3NFs contribute little to the triples corrections.

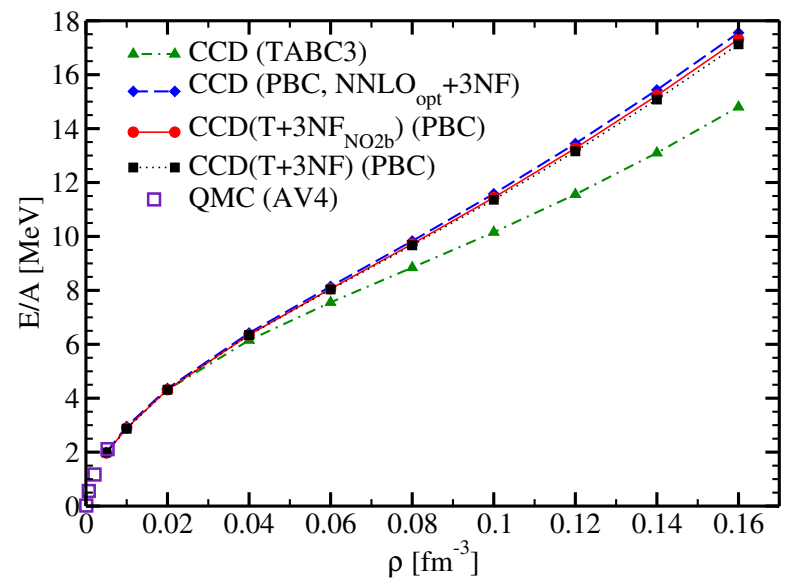

FIG. 10. (Color online) Energy per particle in neutron matter with $\mathrm{NNLO}_{\text {opt }}$ (NN only) and with inclusion of $3 \mathrm{NF}$ computed in the CCD and CCD $(\mathrm{T})$ approximations. The 3NF LECs are given by $c_{E}=-0.389$ and $c_{D}=-0.39$. The calculations used $A=66$ neutrons, $n_{\max }=4$, and PBC and TABC.

\section{B. Nuclear matter}

In this Subsection we perform coupled-cluster calculations of symmetric nuclear matter using chiral $N N$ and 3NF interactions at NNLO. Figure 11 shows the energy per nucleon in symmetric nuclear matter for a wide range of densities computed in MBPT2, the $\mathrm{CCD}_{\text {ladd }}$, and in the CCD approximation with the $N N$ potential $\mathrm{NNLO}_{\text {opt }}$. In these calculations we used $A=132 \mathrm{nu}-$ cleons, $n_{\max }=4$, and TABC based on $3^{3}$ angles. We observe that the saturation point is at a too large density, and we get a considerable overbinding. These results for $\mathrm{NNLO}_{\mathrm{opt}}$ are in good agreement with the recent self-consistent Green's function (SCGF) calculations of nuclear matter [34], and the $\mathrm{CCD}_{\text {ladd }}$ calculations of Ref. 67]. The difference between MBPT2 and CCD is considerable, indicating that nuclear matter for the $\mathrm{NNLO}_{\text {opt }}$ chiral interaction is not perturbative. The difference between the $\mathrm{CCD}_{\text {ladd }}$ approximation and the full CCD calculations is around $1 \mathrm{MeV}$ per nucleon around saturation density. We can conclude that - in contrast to neutron matter - for nuclear matter and the $\mathrm{NNLO}_{\mathrm{opt}}$ interaction (which is rather soft), non-linear terms in the $T_{2}$ amplitude and particle-hole excitations yield nonnegligible contributions. We note also that the coupledcluster calculations are difficult to converge for Fermi momenta smaller than about $0.8 \mathrm{fm}^{-1}$. This is presumably due to the clustering of nuclear matter at low densities [77.

Let us turn to 3NFs. Figure 12 shows the energy per nucleon in symmetric nuclear matter for a wide range of densities computed with MBPT2, CCD,

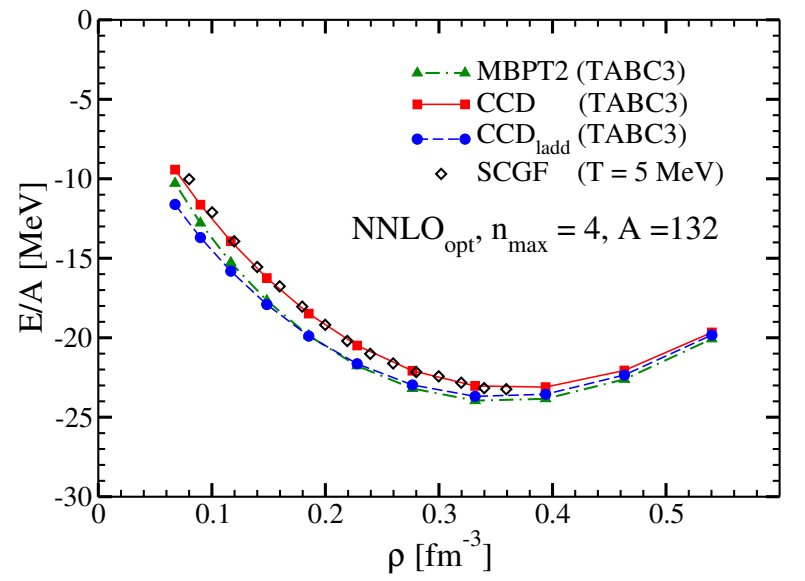

FIG. 11. (Color online) Energy per particle in symmetric nuclear matter with $\mathrm{NNLO}_{\text {opt }}$ (NN only) computed in the MBPT2 (triangles with green dashed-dotted line), $\mathrm{CCD}_{\text {ladd }}$ (circles with blue dashed line), and CCD (squares with solid red line) approximations. The calculations used 132 nucleons, $n_{\max }=4$, and PBC. Diamonds are results from selfconsistent Green's function (SCGF) at the finite temperature $T=5 \mathrm{MeV}$, taken from Ref. 34.

and the $\mathrm{CCD}(\mathrm{T})$ approximation. The $\mathrm{CCD}$ calculations included the $3 \mathrm{NF}$ in the normal-ordered twobody approximation. The $\operatorname{CCD}(\mathrm{T})$ calculations were performed with $3 \mathrm{NFs}$ in the normal-ordered two-body approximation $\left(\mathrm{CCD}\left(\mathrm{T}+3 \mathrm{NF}_{\mathrm{NO}} \mathrm{b}\right)\right)$, and going beyond the normal-ordered two-body approximation by including the leading-order residual $3 \mathrm{NF}$ contribution to the perturbative estimate for the $T_{3}$ amplitude $\left(\mathrm{CCD}\left(\mathrm{T}+3 \mathrm{NF}_{\mathrm{NO} 3 \mathrm{~b}}\right)\right)$. In these calculations we used $A=132$ nucleons with $\mathrm{PBC}$ and $n_{\max }=4$. For the densities we consider here, the difference between $\mathrm{PBC}$ and TABC is small.

In contrast to calculations of neutron matter, the contribution from the perturbative triples corrections is sizable in nuclear matter, and about $1 \mathrm{MeV}$ per nucleon in the range of densities shown when including the $3 \mathrm{NF}$ in the normal-ordered two-body approximation. Furthermore, we find that the contribution of the residual $3 \mathrm{NF}$ to the $\mathrm{CCD}(\mathrm{T})$ energy is significant around saturation density, indicating that the normal-ordered twobody approximation for the $3 \mathrm{NF}$ might not be sufficient in symmetric nuclear matter. We checked that the contribution of the residual 3NF to the CCD amplitude equations is negligible, and therefore it might be sufficient to include the full $3 \mathrm{NF}$ in the perturbative triples amplitude. In order to check the accuracy of the perturbative triples approximation $(\mathrm{CCD}(\mathrm{T}))$ in nuclear matter we also performed non-perturbative, iterative CCDT-1 (see Refs. [78, 79]) calculations for $A=28$ and $n_{\max }=3$ at two different densities $k_{F}=1.3 \mathrm{fm}^{-1}$ and $k_{F}=1.6 \mathrm{fm}^{-1}$. We found that the difference between $\mathrm{CCD}(\mathrm{T})$ and $\mathrm{CCDT}-1$ in this range of densities is at most $0.1 \mathrm{MeV}$ per nucleon. Therefore, we conclude that the $\mathrm{CCD}(\mathrm{T})$ approximation is accurate for the $N N$ 


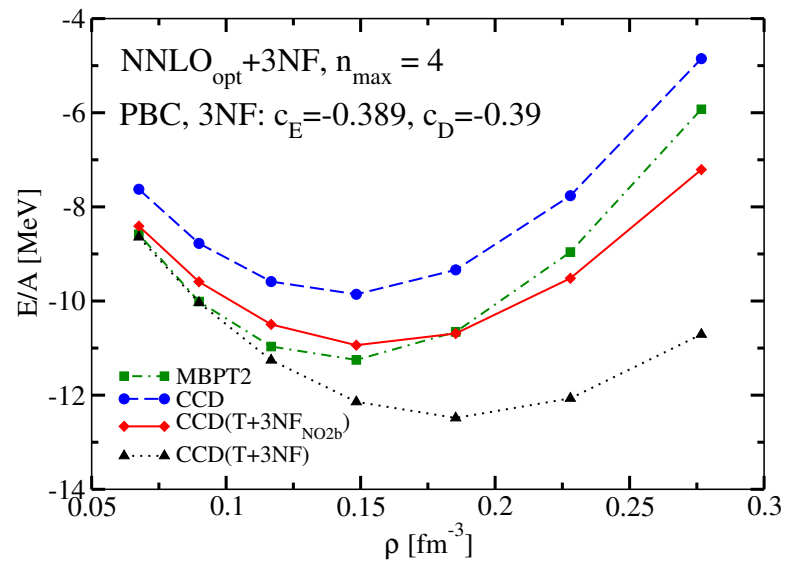

FIG. 12. (Color online) Energy per particle in symmetric nuclear matter with $\mathrm{NNLO}_{\mathrm{opt}}$ and $3 \mathrm{NF}$ computed in the MBPT2 (squares with green dashed-dotted line), CCD (circles with blue dashed line), and $\operatorname{CCD}(\mathrm{T})$ with $3 \mathrm{NF}$ in the normal-ordered two-body approximation (diamonds with solid red line), and including the residual $3 \mathrm{NF}$ in leading order (triangles with dotted black line). The 3NF LECs are given by $c_{E}=-0.389$ and $c_{D}=-0.39$. The calculations used 132 nucleons, $n_{\max }=4$, and PBC.

potential $\mathrm{NNLO}_{\text {opt }}$ and chiral $3 \mathrm{NFs}$ in symmetric nuclear matter.

\section{Scheme dependence of three-nucleon forces}

In this Subsection, we try to further illuminate the role of 3NFs in nucleonic matter. We study different regularization schemes, and compute the energy per particle in pure neutron matter and symmetric nuclear matter. The 3NF employed in the previous Subsections exhibits a cutoff of $\Lambda=500 \mathrm{MeV}$. This cutoff is in the momentum transfer, and therefore local in position space 60. This choice of regulator for the $3 \mathrm{NF}$ is different from the regularization scheme that is used in the nucleon-nucleon sector, and from other regularizations of the $3 \mathrm{NF}$ that exhibit cutoffs on Jacobi momenta [38. We note that regulators that cut off initial and final Jacobi momenta lead to non-local interactions. Here, the cutoff function is

$$
f_{R}(\vec{p}, \vec{q})=\exp \left[-\left(\frac{4 p^{2}+3 q^{2}}{4 \Lambda^{2}}\right)^{n}\right]
$$

with $\vec{p}=\left(\vec{k}_{1}-\vec{k}_{2}\right) / 2$ and $q=\left[\vec{k}_{3}-\left(\vec{k}_{1}-\vec{k}_{2}\right) / 2\right](2 / 3)$. This regulator reduces to the regulator used in the $N N$ sector for $\vec{q}=0$. In the $N N$ potential $\mathrm{NNLO}_{\mathrm{opt}}$ we use $n=3$, while for the local regulator of the $3 \mathrm{NF}$ defined in Ref. 60 we use $n=2$ in the exponential. In what follows, we compare the $\mathrm{NNLO}_{\text {opt }}$ interaction with a $3 \mathrm{NF}$ that also uses a local regulator but a lower cutoff of $\Lambda=400 \mathrm{MeV}$, and with a $3 \mathrm{NF}$ that employs a nonlocal regulator and a cutoff $\Lambda=500 \mathrm{MeV}$ in relative Jacobi momenta.
Figure 13 shows the energy per particle in pure neutron matter computed in the $\operatorname{CCD}(\mathrm{T})$ approximation. Here we included $3 \mathrm{NFs}$ in the normal ordered two-body approximation, and in the $\mathrm{CCD}\left(\mathrm{T}: w T_{2}=0\right)$ approximation. For the latter, we went beyond the normal ordered two-body approximation and included the residual threebody term $w$ that enters at first order in the triples equation for $T_{3}$. In neutron matter the contribution from the residual $3 \mathrm{NF} w$ to the energy per particle is small. This indicates that the normal-ordered two-body approximation works very well. In the EoS calculation with the local regulator and the lower cutoff $\Lambda=400 \mathrm{MeV}$ we adjusted the LECs of the three-body contact term to $c_{E}=-0.27$ and kept $c_{D}$ unchanged. Then, the binding energies of the triton and the nuclei ${ }^{3,4} \mathrm{He}$ are close to the experimental values. For the non-local regulator with cutoff $\Lambda=500$ and power $n=2$ in the exponential, the LECs $c_{E}=-0.791$ and $c_{D}=-2$ reproduce the triton and ${ }^{3} \mathrm{He}$ binding energies. In pure neutron matter the contributions from the $3 \mathrm{NF}$ contact terms with the LECs $c_{E}$ and $c_{D}$ vanish for a non-local regulator, and the contribution to the EoS depends only on the pion-nucleon couplings $c_{1}$ and $c_{3}$ of the long-range two-pion exchange term of the $3 \mathrm{NF}$ 40. However, for a local regulator the $3 \mathrm{NF}$ contact terms do not vanish in neutron matter [29]. The results for the EoS for pure neutron matter show a regulator dependence at densities beyond $\rho=0.08 \mathrm{fm}^{-3}$. The band obtained from the different $3 \mathrm{NF}$ regulators are within the corresponding band for neutron matter obtained in Ref. [44].

Figure 14 shows the corresponding plot for the energy per particle in symmetric nuclear matter. Here the results for the local regulator with a cutoff $\Lambda=500 \mathrm{MeV}$ exhibit a considerable enhancement of the contribution from the residual 3NF $w$ to the energy per particle at densities above the saturation densities. The sizeable triples contribution of the residual $3 \mathrm{NF} w$ questions the usually observed hierarchy of the coupled-cluster approximation. The results from the lower cutoff $\Lambda=400 \mathrm{MeV}$ are much more satisfactory in the sense that the contribution from the residual three-body part $w$ to the binding energy per particle is considerably smaller, and at the order of $0.5 \mathrm{MeV}$ or less for the densities considered. Likewise, the results obtained with the non-local regulator at the cutoff $500 \mathrm{MeV}$ are also satisfactory in the sense that the contribution from the residual $3 \mathrm{NF} w$ is at most $1 \mathrm{MeV}$ to the energy per particle at densities beyond the saturation point. One might speculate whether this problematic feature of the local regulator with a cutoff $500 \mathrm{MeV}$ is related to the large cutoff dependence found in finite nuclei using this regulator 64. Naively one would expect that regulator dependencies are higher-order corrections in an EFT. The large scheme dependencies observed in Fig. 14 might therefore suggest that the cutoff $\Lambda=500 \mathrm{MeV}$ is too close to the EFT breakdown scale.

For local and non-local regulators we considerably underbind nuclear matter. The saturation density for the local regulators is too high, while for the non-local regula- 


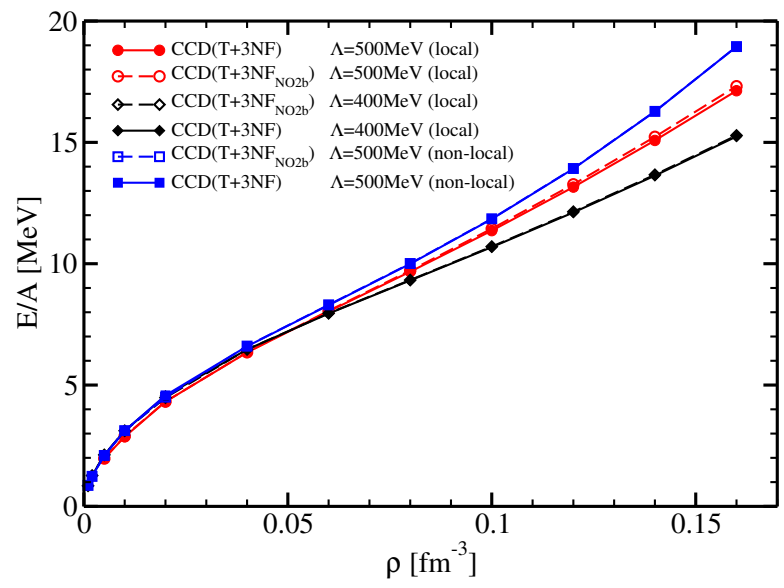

FIG. 13. (Color online) Energy per particle in pure neutron matter with $\mathrm{NNLO}_{\text {opt }}$ and $3 \mathrm{NF}$ computed in the $\mathrm{CCD}(\mathrm{T})$ approximation including $3 \mathrm{NFs}$ in the normal ordered twobody approximation and including the residual $3 \mathrm{NF}$ in the $\mathrm{CCD}\left(\mathrm{T}: w T_{2}=0\right)$ approximation. For the $3 \mathrm{NF}$ we used a local regulator with cutoffs $\Lambda=400$ and $\Lambda=500 \mathrm{MeV}$. The 3NF LECs are given by $c_{E}=-0.389$ and $c_{D}=-0.39$ for the $\Lambda=500 \mathrm{MeV}$ local regulator, while for the $\Lambda=400 \mathrm{MeV}$ local regulator we used $c_{E}=-0.27$ and $c_{D}=-0.39$ with $c_{E}$ adjusted to the ${ }^{4} \mathrm{He}$ binding energy. For the non-local regulator with $\Lambda=500 \mathrm{MeV}$ cutoff we used $c_{E}=-0.791$ and $c_{D}=-2$ adjusted to the triton and ${ }^{3} \mathrm{He}$ binding energies. The calculations used 66 neutrons, $n_{\max }=4$, and PBC

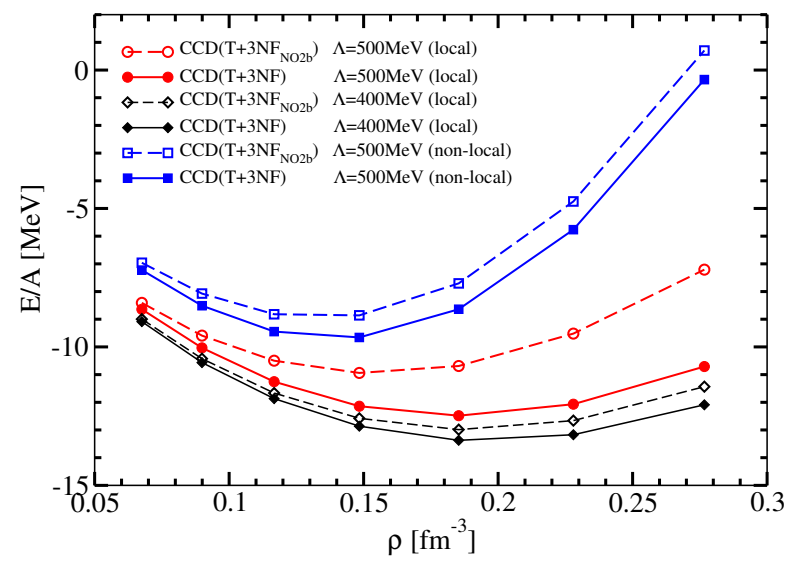

FIG. 14. (Color online) Same caption as in Fig. 13 , except that the energy per particle is for symmetric nuclear matter. The calculations used 132 nucleons, $n_{\max }=4$, and PBC

tor the saturation density is closer to the empirical value. We tried to adjust the LECs $c_{E}$ and $c_{D}$ such that an acceptable result could be obtained simultaneously for the saturation point in symmetric nuclear matter and the triton binding energy. For the non-local regulator the result is shown in Fig. 15. The blue band shows the region where the triton binding energy is reproduced within $5 \%$. The red band shows the region where the saturation Fermi momentum is within $5 \%$ of its empirical value, and the green band shows the region where the energy per nu- cleon is within $5 \%$ of the empirical value. The nuclear matter calculations were obtained from MBPT2 calculations using 28 nucleons, and we accounted for about $1 \mathrm{MeV}$ per nucleon in missing correlations energy, and about $0.5 \mathrm{MeV}$ per nucleon due to finite size effects. It thus seems that a simultaneous reproduction of saturation in light nuclei and infinite matter is not possible without adjusting other LECs. As an example we considered the point $c_{E}=0.3$ and $c_{D}=-2.0$. This yields the saturation point $k_{F} \approx 1.4 \mathrm{fm}^{-1}$ and $E / A \approx 15.5 \mathrm{MeV}$, while the triton binding energy is $-13.53 \mathrm{MeV}$.

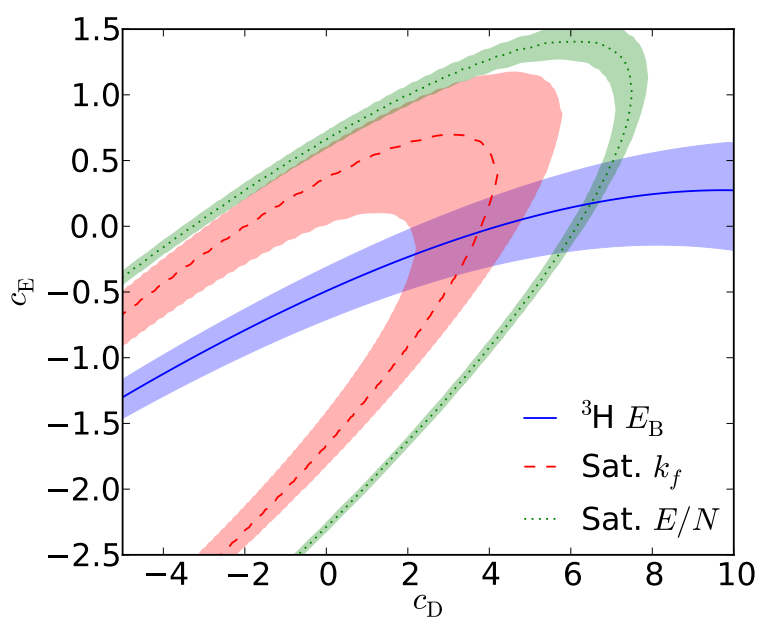

FIG. 15. (Color online) The blue band shows the region where the triton binding energy is reproduced within $5 \%$ of the experimental value. The red band shows the region where the saturation fermi momentum in symmetric nuclear matter is reproduced within $5 \%$ of its empirical value, and the green band shows the region where the energy per nucleon is within $5 \%$ of the empirical value.

We would like to understand better the role that different regulators and cutoffs play for the chiral 3NF. Unfortunately, it is difficult to visualize 3NFs in momentum space [80, 81. We therefore compute the MBPT2 contribution of the residual 3NF $w$ and cut off the involved momentum integrations at a single-particle momentum $k_{\text {cut }}$. Figure 16 shows the fractional contribution of the MBPT2 energy correction of the residual $3 \mathrm{NF}$ as a function of $k_{\text {cut }}$ at the Fermi momentum $k_{F}=1.3 \mathrm{fm}^{-1}$. The chiral cutoff of $\Lambda=500 \mathrm{MeV}$ is also shown as a dashed line for comparison. We see that for the local cutoff $\Lambda=500 \mathrm{MeV}$ most contributions to the MBPT2 result are from high single-particle momenta that are well above the nominal chiral cutoff. The situation is improved for the local regulator with lower cutoff $\Lambda=400 \mathrm{MeV}$ and even more so for the nonlocal regulator with cutoff $\Lambda=500 \mathrm{MeV}$. For a discussion of different cutoff schemes and convergence issues in calculations of the homogeneous electron gas see Ref. [51].

Let us finally note that issues with $3 \mathrm{NF}$ s also arose in other calculations. Lovato et al. 29] pointed out that the equivalence of different chiral $3 \mathrm{NF}$ contact terms 38 is 


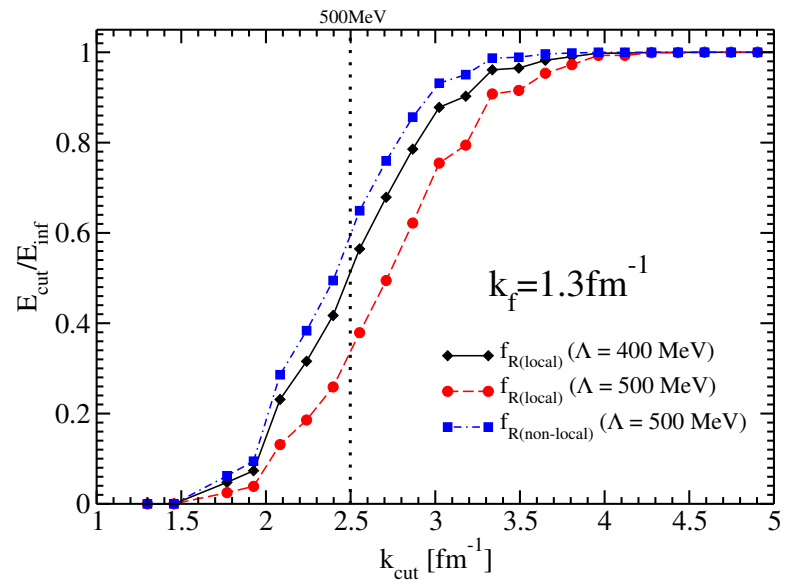

FIG. 16. (Color online) Cutoff dependent fraction of the residual 3NF contribution to the MBPT2 energy per particle in symmetric nuclear matter for different regulators. Results are shown for the local regulator with $\Lambda=500 \mathrm{MeV}$ (diamonds), the local regulator with $\Lambda=400 \mathrm{MeV}$ (squares), and for the non-local regulator with $\Lambda=500 \mathrm{MeV}$ (circles). Calculations used 132 nucleons, $n_{\max }=4$, and PBC.

spoiled by local regulators. Roth et al. 82] used SRG evolution to soften the chiral $N N$ interaction of Ref. 83. combined with the local $3 \mathrm{NF}$ of Ref. [60], and found that the results in medium-mass nuclei depend considerably on the SRG evolution scale. This dependence is reduced for a cutoff $\Lambda=400 \mathrm{MeV}$ in the local 3NF 64. Clearly, more studies of chiral 3NFs are necessary to fully understand regularization scheme dependences.

\section{SUMMARY}

We have performed coupled-cluster calculations of nucleonic matter with interactions from chiral EFT at NNLO. The single-particle states consist of a discrete lattice in momentum space, and the implementation of twist-averaged boundary conditions mitigates shell oscillations and finite-size effects. Our benchmark calculations agree well with other well-established methods. We find that neutron matter is perturbative, while symmetric nuclear matter is not perturbative, with significant contributions beyond perturbation theory and particle ladders.

For the employed $N N$ potential $\mathrm{NNLO}_{\text {opt }}$ and $3 \mathrm{NFs}$, the neutron matter results fall within the error estimates of previous calculations for chiral interactions, with 3NFs acting repulsively. For nuclear matter, the empirical saturation could not be reproduced, and the results are very sensitive to the employed regulator (local vs. nonlocal) and cutoff. At larger chiral cutoffs, the nonlocal regulator is preferred over the local one because it corresponds closer to the cutoff generated by the finite single-particle basis. It seems that the variation of the $3 \mathrm{NF}$ contact terms alone is insufficient to achieve both an acceptable saturation point of nuclear matter and an acceptable binding of light nuclei.

\section{ACKNOWLEDGMENTS}

We thank S. K. Bogner, E. Epelbaum, R. J. Furnstahl, A. Mukherjee, and F. Pederiva for discussions. This work was supported by the Office of Nuclear Physics, U.S. Department of Energy (Oak Ridge National Laboratory), under DE-FG02-96ER40963 (University of Tennessee), DE-FG02-87ER40365 (Indiana University), desc0008499 and de-sc0008808 (NUCLEI SciDAC collaboration), the Field Work Proposal ERKBP57 at Oak Ridge National Laboratory, the LDRD program at Los Alamos National Laboratory, and the Research Council of Norway under contract ISP-Fysikk/216699. Computer time was provided by the Innovative and Novel Computational Impact on Theory and Experiment (INCITE) program. This research used resources of the Oak Ridge Leadership Computing Facility located in the Oak Ridge National Laboratory, which is supported by the Office of Science of the Department of Energy under Contract No. DE-AC05-00OR22725, and used computational resources of the National Center for Computational Sciences, the National Institute for Computational Sciences, and the Notur project in Norway. Computing time has also been provided by Los Alamos Open Supercomputing. This research also used resources of the National Energy Research Scientific Computing Center, which is supported by the Office of Science of the U.S. Department of Energy under Contract No. DE-AC02-05CH11231.
[1] A. Burrows, Rev. Mod. Phys. 85, 245 (2013), URL http: //link.aps.org/doi/10.1103/RevModPhys.85.245.

[2] F. Weber, Pulsars as Astrophysical Laboratories for $\mathrm{Nu}$ clear and Particle Physics (Institute of Physics Publishing, London, 1999).

[3] H. Heiselberg and M. Hjorth-Jensen, Phys. Rep. 328, 237 (2000).

[4] J. M. Lattimer and M. Prakash, Phys. Rep. 442, 109 (2007).
[5] F. Sammarruca, International Journal of Modern Physics E 19, 1259 (2010), URL http://www .worldscientific. com/doi/abs/10.1142/S0218301310015874.

[6] J. M. Lattimer, Ann. Rev. Nucl. Part. Science 62, 485 (2012).

[7] K. Hebeler, J. M. Lattimer, C. J. Pethick, and A. Schwenk, Astrophys. J. 773, 11 (2013).

[8] B. Alex Brown, Phys. Rev. Lett. 85, 5296 (2000), URL http://link.aps.org/doi/10.1103/PhysRevLett.85. 
5296

[9] C. J. Horowitz and J. Piekarewicz, Phys. Rev. Lett. 86, 5647 (2001), URL http://link.aps.org/doi/10.1103/ PhysRevLett.86.5647

[10] S. Gandolfi, J. Carlson, and S. Reddy, Phys. Rev. C 85, 032801 (2012), URL http://link.aps .org/doi/10. 1103/PhysRevC.85.032801

[11] M. B. Tsang, J. R. Stone, F. Camera, P. Danielewicz, S. Gandolfi, K. Hebeler, C. J. Horowitz, J. Lee, W. G. Lynch, Z. Kohley, et al., Phys. Rev. C 86, 015803 (2012), URL http://link.aps .org/doi/10. 1103/PhysRevC.86.015803

[12] A. W. Steiner and S. Gandolfi, Phys. Rev. Lett. 108, 081102 (2012), URL http://link.aps.org/doi/ 10.1103/PhysRevLett.108.081102

[13] S. Shlomo and D. H. Youngblood, Phys. Rev. C 47, 529 (1993), URL http://link.aps.org/doi/10.1103/ PhysRevC.47.529

[14] S. Abrahamyan, Z. Ahmed, H. Albataineh, K. Aniol, D. S. Armstrong, W. Armstrong, T. Averett, B. Babineau, A. Barbieri, V. Bellini, et al. (PREX Collaboration), Phys. Rev. Lett. 108, 112502 (2012), URL http://link.aps.org/doi/10.1103/PhysRevLett.108. 112502.

[15] P.-G. Reinhard, J. Piekarewicz, W. Nazarewicz, B. K. Agrawal, N. Paar, and X. Roca-Maza, Phys. Rev. C 88, 034325 (2013), URL http://link.aps .org/doi/10. 1103/PhysRevC.88.034325

[16] J. Erler, C. J. Horowitz, W. Nazarewicz, M. Rafalski, and P.-G. Reinhard, Phys. Rev. C 87, 044320 (2013).

[17] M. Kortelainen, T. Lesinski, J. Moré, W. Nazarewicz, J. Sarich, N. Schunck, M. V. Stoitsov, and S. Wild, Phys. Rev. C 82, 024313 (2010), URL http://link.aps.org/ doi/10.1103/PhysRevC.82.024313

[18] D. Lunney, J. M. Pearson, and C. Thibault, Rev. Mod. Phys. 75, 1021 (2003), URL http://link.aps.org/doi/ 10.1103/RevModPhys.75.1021.

[19] B. D. Day, Rev. Mod. Phys. 39, 719 (1967), URL http: //link.aps.org/doi/10.1103/RevModPhys.39.719.

[20] K. A. Brueckner, C. A. Levinson, and H. M. Mahmoud, Phys. Rev. 95, 217 (1954).

[21] K. A. Brueckner, Phys. Rev. 100, 36 (1955).

[22] M. Baldo and G. F. Burgio, Reports on Progress in Physics 75, 026301 (2012).

[23] M. Baldo, A. Polls, A. Rios, H.-J. Schulze, and I. Vidaña, Phys. Rev. C 86, 064001 (2012), URL http://link.aps . org/doi/10.1103/PhysRevC.86.064001.

[24] H. Kümmel, K. H. Lührmann, and J. G. Zabolitzky, Phys. Rep. 36, 1 (1978).

[25] B. D. Day and G. Zabolitzky, Nucl. Phys. A 366, 221 (1981).

[26] J. Carlson, J. Morales, V. R. Pandharipande, and D. G. Ravenhall, Phys. Rev. C 68, 025802 (2003), URL http: //link.aps.org/doi/10.1103/PhysRevC.68.025802

[27] S. Gandolfi, A. Y. Illarionov, K. E. Schmidt, F. Pederiva, and S. Fantoni, Phys. Rev. C 79, 054005 (2009), URL http://link.aps.org/doi/10. 1103/PhysRevC.79.054005

[28] A. Gezerlis and J. Carlson, Phys. Rev. C 81, 025803 (2010), URL http://link.aps.org/doi/10. 1103/PhysRevC.81.025803.

[29] A. Lovato, O. Benhar, S. Fantoni, and K. E. Schmidt, Phys. Rev. C 85, 024003 (2012), URL http://link.aps . org/doi/10.1103/PhysRevC.85.024003.
[30] A. Gezerlis, I. Tews, E. Epelbaum, S. Gandolfi, K. Hebeler, A. Nogga, and A. Schwenk, Phys. Rev. Lett. 111, 032501 (2013), URL http://link.aps.org/doi/ 10.1103/PhysRevLett.111.032501

[31] W. Dickhoff and C. Barbieri, Progress in Particle and Nuclear Physics 52, 377 (2004), URL http://www.sciencedirect.com/science/article/ pii/S0146641004000535

[32] V. Somà and P. Bożek, Phys. Rev. C 78, 054003 (2008), URL http://link.aps.org/doi/10.1103/PhysRevC. 78.054003

[33] A. Rios and V. Somà, Phys. Rev. Lett. 108, 012501 (2012), URL http://link.aps.org/doi/10. 1103/PhysRevLett.108.012501

[34] A. Carbone, A. Polls, and A. Rios, Phys. Rev. C 88, 044302 (2013).

[35] R. Machleidt and D. Entem, Physics Reports 503, 1 (2011), ISSN 0370-1573, URL http://www.sciencedirect.com/science/article/ pii/S0370157311000457

[36] E. Epelbaum, H.-W. Hammer, and U.-G. Meißner, Rev. Mod. Phys. 81, 1773 (2009), URL http://link.aps. org/doi/10.1103/RevModPhys.81.1773.

[37] U. van Kolck, Phys. Rev. C 49, 2932 (1994), URL http: //link.aps.org/doi/10.1103/PhysRevC.49.2932.

[38] E. Epelbaum, A. Nogga, W. Glöckle, H. Kamada, U.-G. Meißner, and H. Witała, Phys. Rev. C 66, 064001 (2002), URL http://link.aps.org/doi/10. 1103/PhysRevC.66.064001

[39] E. Epelbaum, H. Krebs, D. Lee, and U.-G. Meißner, The European Physical Journal A 40, 199 (2009), ISSN 1434-6001, URL http://dx.doi.org/10.1140/ epja/i2009-10755-0.

[40] K. Hebeler and A. Schwenk, Phys. Rev. C 82, 014314 (2010), URL http://link.aps.org/doi/10. 1103/PhysRevC.82.014314.

[41] K. Hebeler, S. K. Bogner, R. J. Furnstahl, A. Nogga, and A. Schwenk, Phys. Rev. C 83, 031301 (2011), URL http: //link.aps.org/doi/10.1103/PhysRevC.83.031301.

[42] J. Holt, N. Kaiser, and W. Weise, Nuclear Physics A 876, 61 (2012), ISSN 0375-9474, URL http://www.sciencedirect.com/science/article/ pii/S0375947411006701

[43] H.-W. Hammer, A. Nogga, and A. Schwenk, Rev. Mod. Phys. 85, 197 (2013).

[44] T. Krüger, I. Tews, K. Hebeler, and A. Schwenk, Phys. Rev. C 88, 025802 (2013), URL http://link.aps.org/ doi/10.1103/PhysRevC.88.025802

[45] L. Coraggio, J. W. Holt, N. Itaco, R. Machleidt, and F. Sammarruca, Phys. Rev. C 87, 014322 (2013), URL http://link.aps.org/doi/10. 1103/PhysRevC.87.014322

[46] T. Inoue, S. Aoki, T. Doi, T. Hatsuda, Y. Ikeda, N. Ishii, K. Murano, H. Nemura, and K. Sasaki (HAL QCD Collaboration), Phys. Rev. Lett. 111, 112503 (2013).

[47] D. J. Dean and M. Hjorth-Jensen, Phys. Rev. C 69, 054320 (2004), URL http://link.aps .org/doi/10. 1103/PhysRevC.69.054320.

[48] K. Kowalski, D. J. Dean, M. Hjorth-Jensen, T. Papenbrock, and P. Piecuch, Phys. Rev. Lett. 92, 132501 (2004), URL http://link.aps.org/doi/10. 1103/PhysRevLett.92.132501

[49] R. J. Bartlett and M. Musiał, Rev. Mod. Phys. 79, 291 (2007). 
[50] I. Shavitt and R. J. Bartlett, Many-body Methods in Chemistry and Physics (Cambridge University Press, 2009).

[51] J. J. Shepherd, A. Grüneis, G. H. Booth, G. Kresse, and A. Alavi, Phys. Rev. B 86, 035111 (2012), URL http: //link.aps.org/doi/10.1103/PhysRevB.86.035111.

[52] A. Roggero, A. Mukherjee, and F. Pederiva, Phys. Rev. B 88, 115138 (2013), URL http://link.aps.org/doi/ 10.1103/PhysRevB.88.115138.

[53] F. E. Harris, H. J. Monkhorst, and D. L. Freeman, Algebraic and diagrammatic methods in many-fermion theory (Oxford University Press, 1992).

[54] D. L. Freeman, Phys. Rev. B 15, 5512 (1977), URL http: //link.aps.org/doi/10.1103/PhysRevB.15.5512

[55] R. F. Bishop and K. H. Lührmann, Phys. Rev. B 17, 3757 (1978), URL http://link.aps .org/doi/10.1103/ PhysRevB.17.3757

[56] A. Ekström, G. Baardsen, C. Forssén, G. Hagen, M. Hjorth-Jensen, G. R. Jansen, R. Machleidt, W. Nazarewicz, T. Papenbrock, J. Sarich, et al., Phys. Rev. Lett. 110, 192502 (2013), URL http://link.aps . org/doi/10.1103/PhysRevLett.110.192502.

[57] C. Gros, Zeitschrift für Physik B Condensed Matter 86, 359 (1992), ISSN 0722-3277, URL http://dx.doi.org/ 10.1007/BF01323728

[58] C. Gros, Phys. Rev. B 53, 6865 (1996), URL http:// link.aps.org/doi/10.1103/PhysRevB.53.6865.

[59] C. Lin, F. H. Zong, and D. M. Ceperley, Phys. Rev. E 64, 016702 (2001), URL http://link.aps.org/doi/10. 1103/PhysRevE.64.016702

[60] P. Navrátil, Few-Body Systems 41, 117 (2007), ISSN 0177-7963, URL http://dx.doi.org/10.1007/ s00601-007-0193-3.

[61] P. Navratil, S. Quaglioni, and D. Gazit, private communication.

[62] G. Hagen, T. Papenbrock, D. J. Dean, A. Schwenk, A. Nogga, M. Włoch, and P. Piecuch, Phys. Rev. C 76, 034302 (2007), URL http://link.aps .org/doi/10. 1103/PhysRevC.76.034302

[63] S. Binder, P. Piecuch, A. Calci, J. Langhammer, P. Navrátil, and R. Roth, ArXiv e-prints (2013), 1309.1123.

[64] R. Roth, S. Binder, K. Vobig, A. Calci, J. Langhammer, and P. Navrátil, Phys. Rev. Lett. 109, 052501 (2012), URL http://link.aps.org/doi/10. 1103/PhysRevLett.109.052501

[65] G. Hagen, T. Papenbrock, D. J. Dean, and M. HjorthJensen, Phys. Rev. Lett. 101, 092502 (2008), URL http://link.aps.org/doi/10.1103/PhysRevLett.101. 092502 .
[66] G. Hagen, T. Papenbrock, D. J. Dean, and M. HjorthJensen, Phys. Rev. C 82, 034330 (2010), URL http:// link.aps.org/doi/10.1103/PhysRevC.82.034330.

[67] G. Baardsen, A. Ekström, G. Hagen, and M. HjorthJensen, Phys. Rev. C 88, in press (2013).

[68] S. K. Bogner, R. J. Furnstahl, and R. J. Perry, Phys. Rev. C 75, 061001 (2007).

[69] K. Raghavachari, The Journal of Chemical Physics 82, 4607 (1985), URL http://link.aip.org/link/?JCP/ 82/4607/1

[70] B. D. Day, Rev. Mod. Phys. 50, 495 (1978).

[71] B. D. Day, Phys. Rev. C 24, 1203 (1981), URL http: //link.aps.org/doi/10.1103/PhysRevC.24.1203.

[72] M. I. Haftel and F. Tabakin, Nucl. Phys. A 158, 1 (1970).

[73] K. Suzuki, R. Okamoto, M. Kohno, and S. Nagata, Nucl. Phys. A 665, 92 (2000).

[74] K. A. Brueckner and J. L. Gammel, Phys. Rev. 109, 1023 (1958).

[75] K. Schmidt and S. Fantoni, Physics Letters B 446, 99 (1999), ISSN 0370-2693, URL http://www.sciencedirect.com/science/article/ pii/S0370269398015226

[76] D. Thompson, M. Lemere, and Y. Tang, Nuclear Physics A 286, 53 (1977), ISSN 03759474, URL http://www.sciencedirect.com/science/ article/pii/0375947477900070

[77] C. Horowitz and A. Schwenk, Nuclear Physics A 776, 55 (2006), ISSN 0375-9474, URL http://www.sciencedirect.com/science/article/ pii/S0375947406002132

[78] Y. S. Lee and R. J. Bartlett, The Journal of Chemical Physics 80, 4371 (1984), URL http://link.aip.org/ link/? JCP/80/4371/1.

[79] Y. S. Lee, S. A. Kucharski, and R. J. Bartlett, The Journal of Chemical Physics 81, 5906 (1984), URL http: //link.aip.org/link/?JCP/81/5906/1.

[80] K. Hebeler, Phys. Rev. C 85, 021002 (2012), URL http: //link.aps.org/doi/10.1103/PhysRevC.85.021002.

[81] K. A. Wendt, Phys. Rev. C 87, 061001 (2013), URL http://link.aps.org/doi/10.1103/PhysRevC.87. 061001

[82] R. Roth, J. Langhammer, A. Calci, S. Binder, and P. Navrátil, Phys. Rev. Lett. 107, 072501 (2011), URL http://link.aps.org/doi/10.1103/PhysRevLett.107. 072501

[83] D. R. Entem and R. Machleidt, Phys. Rev. C 68, 041001 (2003), URL http://link.aps.org/doi/10. 1103/PhysRevC.68.041001. 